\title{
The Effects of Changes in the Environment on the Spatial Firing of Hippocampal Complex-Spike Cells
}

\author{
Robert U. Muller and John L. Kubie \\ Departments of Physiology and Anatomy and Cell Biology, Downstate Medical Center (SUNY), Brooklyn, New York 11203
}

Using the techniques set out in the preceding paper (Muller et al., 1987), we investigated the response of place cells to changes in the animal's environment. The standard apparatus used was a cylinder, $76 \mathrm{~cm}$ in diameter, with walls 51 $\mathrm{cm}$ high. The interior was uniformly gray except for a white cue card that ran the full height of the wall and occupied $100^{\circ}$ of arc. The floor of the apparatus presented no obstacles to the animal's motions.

Each of these major features of the apparatus was varied while the others were held constant. One set of manipulations involved the cue card. Rotating the cue card produced equal rotations of the firing fields of single cells. Changing the width of the card did not affect the size, shape, or radial position of firing fields, although sometimes the field rotated to a modest extent. Removing the cue card altogether also left the size, shape, and radial positions of firing fields unchanged, but caused fields to rotate to unpredictable angular positions.

The second set of manipulations dealt with the size and shape of the apparatus wall. When the standard (small) cylinder was scaled up in diameter and height by a factor of 2 , the firing fields of $36 \%$ of the cells observed in both cylinders also scaled, in the sense that the field stayed at the same angular position and at the same relative radial position. Of the cells recorded in both cylinders, $52 \%$ showed very different firing patterns in one cylinder than in the other. The remaining $12 \%$ of the cells were virtually silent in both cylinders. Similar results were obtained when individual cells were recorded in both a small and a large rectangular enclosure. By contrast, when the apparatus floor plan was changed from circular to rectangular, the firing pattern of a cell in an apparatus of one shape could not be predicted from a knowledge of the firing pattern in the other shape.

The final manipulations involved placing vertical barriers into the otherwise unobstructed floor of the small cylinder. When an opaque barrier was set up to bisect a previously ecorded firing field, in almost all cases the firing field was

\footnotetext{
Received Mar. 17, 1986; revised Dec. 1, 1986; accepted Jan. 8, 1987.

This work was supported by NIH Grants NS 20686 to J.L.K. and R.U.M. and NS 14496 to Dr. James B. Ranck, Jr. We are indebted to Mr. Bobby Marsh of the Department of Physiology for designing and building the interface that allows us to track the animal's position and record it as a binary number that can be sent to a computer. We thank Dr. James B. Ranck, Jr., for his financial, moral, and intellectual support in the early stages of this work. Without his assistance, this work could not have becn started. We also thank Dr. Stcven E. Fox for his help, and Drs. John O'Keefe and David Zipser for many useful discussions.

Correspondence should be addressed to Dr. Robert Muller, Department of Physiology -Box 31, Downstate Medical Center, 450 Clarkson Avenue, Brooklyn, NY 11203.

Copyright (C) 1987 Society for Neuroscience $0270-6474 / 87 / 071951-18 \$ 02.00 / 0$
}

nearly abolished. This was true even though the barrier occupied only a small fraction of the firing field area. A transparent barrier was effective as the opaque barrier in attenuating firing fields. The lead base used to anchor the vertical barriers did not affect place cell firing. The effectiveness of barriers was confined to their vicinity; a barrier set far from a firing field left it unchanged. In one case, a small firing field expanded when a barrier was set to bisect it, which suggests that the vicinity of the barrier continues to be represented in the place cell population by a "remapping" of the local area onto place cells that were previously inactive or only weakly active in the animal's current surroundings.

In the Discussion, it is argued that place cells respond to the selected environmental manipulations as if they were processing abstract spatial information rather than raw sensory data. It is concluded that the "cognitive mapping" hypothesis of O'Keefe and Nadel (1978) captures an important aspect of the functioning of the hippocampus.

The preceding paper (Muller et al., 1987) was devoted to describing the spatial firing properties of hippocampal place cells in a fixed environment. It was demonstrated that the firing that occurs in delimited portions of the apparatus (firing fields) is location-specific rather than behavior-specific. Once the location-specificity of place cell firing is established, it becomes essential to determine the nature of the sensory information that permits stable firing fields to exist. The work presented here was therefore aimed at investigating changes in firing fields caused by alterations of the animal's environment. The method of determining environmental control over place cell firing is to seek a transformation rule for each environmental manipulation, such that the new spatial firing pattern can be predicted from the pattern in the original situation.

Earlier work on the sensory control of place cell firing focused on several issues. Three studies looked into the degree of control exerted by local versus distant cues. O'Keefe (1976), Olton et al. (1978), and Kubie and Ranck (1983) recorded from place cells as rats ran on elevated, sideless mazes; in each case, the maze was completely. exposed to the laboratory. All 3 studies showed that copies could be substituted for portions of the apparatus without affecting the spatial firing of place cells. Thus, cues proper to the apparatus ("intra-maze" cues) can be ignored by the mapping system. This does not imply that local stimuli never contribute to location-specific firing, but that "extra-maze" cues were prepotent under the conditions employed. A similar conclusion was reached from the effects of rotating an 8-arm maze (Olton et al., 1978; Kubie and Ranck, 1983). Both studies found that firing fields remained fixed relative to the laboratory 
when the maze was rotated to superimpose the new position on the old one.

O'Keefe and Conway (1978) used curtains to isolate a cylindrical region from the rest of the laboratory, in order to create a "controlled cue" environment. They affixed a set of stimuli to the curtains at $90^{\circ}$ intervals and showed that simultaneously rotating the stimuli and the 3 -arm " $T$ " maze by $90^{\circ}$ left firing fields in the same position relative to the cues inside the curtains. This result supports the conclusion derived from rotation experiments in the open laboratory, and also shows that no measurable stimulus control is exerted by cues in a frame larger than the curtained region. If the asymmetrical $\mathrm{T}$ maze was rotated relative to the wall cues, firing often ceased or became spatially homogeneous. O'Keefe and Conway (1978) also tested the effects of deleting one or more of the wall cues. They found that no single stimulus in the set of 4 was required for reliable spatial firing, and that firing was often undisturbed if any 2 of the cues were removed. By contrast, when all 4 cues were taken away, firing usually became spatially homogeneous, often with an overall increase in rate. O'Keefe and Conway (1978) used a wide variety of stimulus types, which allowed them to argue that sensory control over place cell activity is multimodal. A similar conclusion can be drawn from the work of Hill and Best (1981) on the firing of place cells in deafened and blindfolded rats.

Another question about place cells concerns the changes in the firing of individual cells when the rat is put into very different situations. Ranck (1973), O'Keefe and Conway (1978), and Kubie and Ranck (1983) all found that knowing a cell's firing pattern in one situation did not help to predict the pattern in others. Kubie and Ranck (1983) showed that the location of a firing field in 1 of 3 apparatuses had no discernible relationship to the location (or existence) of firing fields in the others. This independence obtained despite the fact that the open apparatus (an elevated 8-arm maze) was the platform for the 2 closed apparatuses (an operant chamber and the rat's home cage), each of which had one transparent wall and no roof. Thus, spatial firing patterns shifted beyond recognition, even though a common part of the laboratory was accessible to the animal in all 3 situations, and cven though part of the visual world was the same in all 3 cases. Kubie and Ranck (1983) also found that rotations of the closed chambers were associated with equal rotations of firing fields in the laboratory frame, or, in other words, that firing was controlled by intra-maze cues.

In addition to its use of automatic data-collection methods, the present work on stimulus control over place cell firing departs from earlier studies in 2 ways. First, the rats' behavioral task of chasing randomly scattered food pellets was the same during all recordings. This minimizes the possibility that changes in spatial firing patterns are due to differences in the animal's activity. Second, recordings were made in a set of very simple environments that were variations of a "standard" apparatus. The standard recording chamber was a gray cylinder, $76 \mathrm{~cm}$ in diameter and $51 \mathrm{~cm}$ high, whose floor was free of obstructions. On the wall was a white cardboard sheet that occupied $100^{\circ}$ of the cylinder's arc, centered at 3 o'clock, as viewed with an overhead TV camera. Some of the major features of this "small cylinder" were independently tested for control over place cell firing. The first class of manipulations included rotating the white cue card, changing its size, and removing it from the small cylinder. The second class included changing the dimensions of the cylinder and using apparatuses of a different shape (rect- angular boxes). Finally, the interior space of the cylinder was made more complicated by placing vertical barriers onto the floor at various positions. The effect of each manipulation was tested by comparing, for individual place cells, the spatial firing pattern in the standard apparatus to the pattern seen in the altered apparatus. Transformation rules for particular manipulations were initially derived by inspection of color-coded plots of firing rate as a function of the animal's position. In most cases, more formal means of demonstrating the validity of the rule were then used.

In the Discussion, the empirical transformation rules will be compared to the firing field changes predicted by an explicit, computational model of place cell firing proposed by Zipser (1985). Zipser's model is compatible with most of the empirical rules, but fails to account for several others. On the other hand, all of the transformations of firing fields produced by the selected manipulations of the environment are consistent with the general notion that the rat hippocampus is directly involved in processing information about the spatial properties of the animal's surroundings (O'Keefe and Nadel, 1978).

Before we examine the data, it would be best to first address an important issue. In recording from the hippocampus under varying conditions, the issues of learning, memory, and plasticity are bound to arise. Our purpose, however, was to look for relationships between the environment and place cell firing in the absence of potentially confounding changes in firing associated with learning. Rats were therefore extensively pretrained in the pellet-chasing task, with the aim of having learning completed before recordings were made. In practice, we find that the spatial firing patterns of single cells in the standard apparatus are generally the same before and after the rat is run in a variant, no matter how different the firing pattern was in the other apparatus. In other words, under the circumstances employed, each environmental manipulation appeared to have an effect only for its tenure. In addition to simplifying interpretation of the effects of altering the environment, the reversibility of firing pattern changes simplifies data gathering because the sequence of manipulations is not critical. We have taken advantage of this property of place cells by trying several environmental changes on individual animals, often whilc recording from the same cell. It should also be noted that this great stability of place cell firing constitutes an excellent baseline from which to look for changes in place cell firing that parallel learning processes.

\section{Materials and Methods}

The methods used were substantially the same as described in Muller et al. (1987), which gives detailed descriptions of training, surgery, electrode construction, single-unit recording, and rat-tracking procedures. This section gives procedures relevant for experiments in which individual units were sequentially recorded under different environmental conditions.

\section{General methods}

Recording chambers. Four principal chambers were used; each was painted gray and had a piece of white cardboard covering part of its wall from top to bottom. For each chamber, whether circular or rectangular in floor plan, this "cue card" was ordinarily centered at 3 o'clock in the fixed TV field. Thus, with the exception of rotation and cardremoval experiments, the cue card was on the right of the firing rate maps used to summarize the activity of place cells. In each map, a line was used to indicate the position of the cue card; the line was omitted if the cue card was not present.

The four principal apparatuses were the small and large cylinders and the small and large rectangles. The small cylinder was $76 \mathrm{~cm}$ in diameter 
and $51 \mathrm{~cm}$ high; the large cylinder was $152 \mathrm{~cm}$ in diameter and $102 \mathrm{~cm}$ high. Thus, the large cylinder was a scaled-by- 2 version of the small one. In each cylinder, the cue card covered $100^{\circ}$ of internal arc. The small rectangle was $40 \times 56 \mathrm{~cm}$ and $51 \mathrm{~cm}$ high; the large rectangle was again a scaled-by- 2 version of the small. In each rectangle, the cue card covered one of the shorter walls. In addition to these apparatuses, a replica of the small cylinder was also used. During recording sessions, the apparatus was surrounded with a circular curtain to control the visual environment.

Rats. Units in this study were recorded from 42 young Long-Evans female rats. Each rat was trained to chase food pellets in each of the 4 principal apparatuses for $15-30 \mathrm{~min} / \mathrm{d}$. It generally took 2 weeks of training before the rat would run around freely in the apparatus in pursuit of food. Following training, a microelectrode bundle was implanted above the dorsal CA1 layer of the hippocampus. Three to five days of recovery were allowed before recordings were made.

Unit selection. The 10 electrodes in each rat were checked several times a day while the rat was in the small cylinder and small rectangle. Cells selected for analysis were well-discriminated complex-spike cells that showed clear location-specific firing in either or both of the screening apparatuses. More precisely, a cell's firing rate in the center of the apparent field had to be greater than about 5 action potentials (AP)/sec. Although the judgment of whether there was a good field was subjective, we invariably found that our estimate of a field's existence and position was corroborated by automatically generated firing rate maps from formal recording sessions. Once a unit was selected, the rat was returned to its home cage for half an hour and the wire was then rechecked. If the unit remained stable, formal recording sessions were run. The data presented here are based on observations of 160 units selected in this way. The cells investigated in detail were, thus, not a random sample of the complex-spike cells encountered during electrode passes. It is our impression that about $65 \%$ of well-isolated complex-spike units behaved as place cells, but a proper estimate will require additional work.

Sessions were run about once per hour; the floor paper was replaced between each pair of sessions. In general, the initial session for a cell was done in one of the small, unaltered apparatuses. These "standard" sessions were $16 \mathrm{~min}$ in duration. Moreover, almost all sessions in the large apparatuses were also $16 \mathrm{~min}$ long, to minimize the fraction of the area unvisited by the animal. By contrast, sessions done after manipulations of the small apparatuses were often $8 \mathrm{~min}$ long, which was sufficient to get a good first impression (by inspection of firing rate maps) of the location, size, and shape of firing fields (see Muller et al., 1987, Fig. 3).

Two factors limited the number of recording sessions that could be run for a given unit. The most common difficulty was the rat's behavior; rats will not chase food pellets for more than a few hours per day. Usually, when a rat was sated, it would crouch near the apparatus wall and spend most of its time grooming. Under these circumstances, firing could not be adequately sampled throughout the apparatus and no further sessions were attempted. If the unit was discriminable the next day, additional sessions could be run. Less often, the waveform of the target unit changed sufficiently during a set of sessions to no longer be discriminable from other units, or to make it unclear whether the same unit was being recorded. If there was a serious question of unit identity because a unit was nearly silent in an apparatus, at the end of the session the animal was transferred to an apparatus in which clear spatial firing had previously been seen. The spike waveform and the location of the firing field were compared to the earlier data. If these were the same, the series of sessions was continued. Changes in spike amplitude or waveform usually happened while connecting and disconnecting the recording cable from the animal's headstage.

The procedures outlined above apply to unit screening and testing for all of the experiments described here. There were some differences, however, if a series of sessions was planned that involved only cylindrical apparatuses, as opposed to a series of sessions in the 4 principal chambers.

\section{Methods for experiments run exclusively in cylinders}

Experiments run only in cylindrical apparatuses included rotations, the use of narrow cue cards (occupying $50^{\circ}$ instead of $100^{\circ}$ of arc), cue-card removals, and the use of barriers. For a unit to be included in any of these studies, it had to exhibit clear spatial firing in the small cylinder during screening. For a cell to be used in a set of rotation experiments, its firing field also had to be away from the center of the cylinder.

Rotation, narrow-card, and card-removal experiments were done in either the small cylinder or its replica. To enable accurate positioning of the cylinder, a set of marks $90^{\circ}$ apart on the outside of the cylinder were matched with arrows painted on the floor of the recording room. The position of the cylinder was verified by locating a calibration light on the cylinder with the TV spot follower. Precautions were taken to minimize information available to the animal about the orientation of the apparatus relative to the laboratory frame during rotation, narrowcard, and card-removal experiments. For such experiments, the animal was carried at an arbitrary angle through the circular curtain before being placed in the chamber, and during the session the experimenter would slowly circle the curtains while throwing in the food pellets. The sequence for an all-cylinder series always began with a standard session in the small cylinder. Thereafter, standard sessions were usually repeated after 1 or 2 altered sessions.

\section{Methods for "4-apparatus" experiments}

These experiments involved recording the location-specific firing of an individual place cell in each of the 4 principal apparatuses. A unit was included in the 4-apparatus sample if, during screening, it showed a firing field in the small cylinder, the small rectangle, or both. We used several sequences for recording in the 4 apparatuses, but the most common was: small cylinder, small rectangle, large cylinder, large rectangle. For each session of a 4-apparatus experiment, the white cue card was centered at 3 o'clock.

\section{Methods for barrier experiments}

In addition to the experiments described above, we ran experiments that involved introducing a barrier into the small cylinder. Two barriers were used. The opaque barrier was a $23 \times 23 \mathrm{~cm}$ piece of 4 -mm-thick fiberboard. It was kept in an upright position by forcing it into a groove in a lead base that was $23 \mathrm{~cm}$ long, $2 \mathrm{~cm}$ high, and $3.5 \mathrm{~cm}$ wide. The base provided enough stability for a rat to climb onto the barrier without tipping it over. A transparent (Plexiglas) barrier of the same dimensions was also used.

One of the barrier manipulations was to place a barrier (or just the lead base) so that it bisected the region of a previously recorded firing field. Initially, the barrier was positioned by looking at printed colorcoded maps of the standard session. In later barrier experiments, maps drawn on a color terminal were used. A cursor was placed on the end pixels of a bisector of the field, and the desired coordinates of the endpoints of the barrier were read directly.

\section{Results}

\section{Manipulations of the cue card}

Cue-card rotations. Although the white card is the only intentional stimulus breaking the radial symmetry of the cylinder, there is no guarantee that it plays a role in supporting the spatial firing of place cells. A way of testing its importance is to change the angular position of the card relative to the laboratory frame; if the card is salient, the firing fields of at least some cells should rotate equally. The outcome of a typical rotation experiment is shown in Figure 1. In Figure $1 A$, the card was in its standard position at 3 o'clock, as indicated by the circular arc. A session was then run with the wall and card both rotated $90^{\circ}$ so that the card was centered at 12 o'clock; in response, the firing field rotated approximately $90^{\circ}$ counterclockwise (Fig. $1 \mathrm{~B}$ ). The same result was obtained 14 out of the 15 times this experiment was repeated, independent of whether the field happened to be near or far from the cue card. For this cell and 10 of 11 others, when the wall and card were returned to their initial position, the field rotated back (Fig. 1C). One cell ceased to fire when the cylinder was rotated and took up a different firing pattern when a second standard session was run; this cell will be mentioned again at the end of this section.

In the session shown in Figure $1 B$, the entire cylinder (wall plus card) was rotated, leaving open the possibility that the angular position of the field followed some feature of the cylinder other than the cue card. In the experiment in Figure 2, only the 

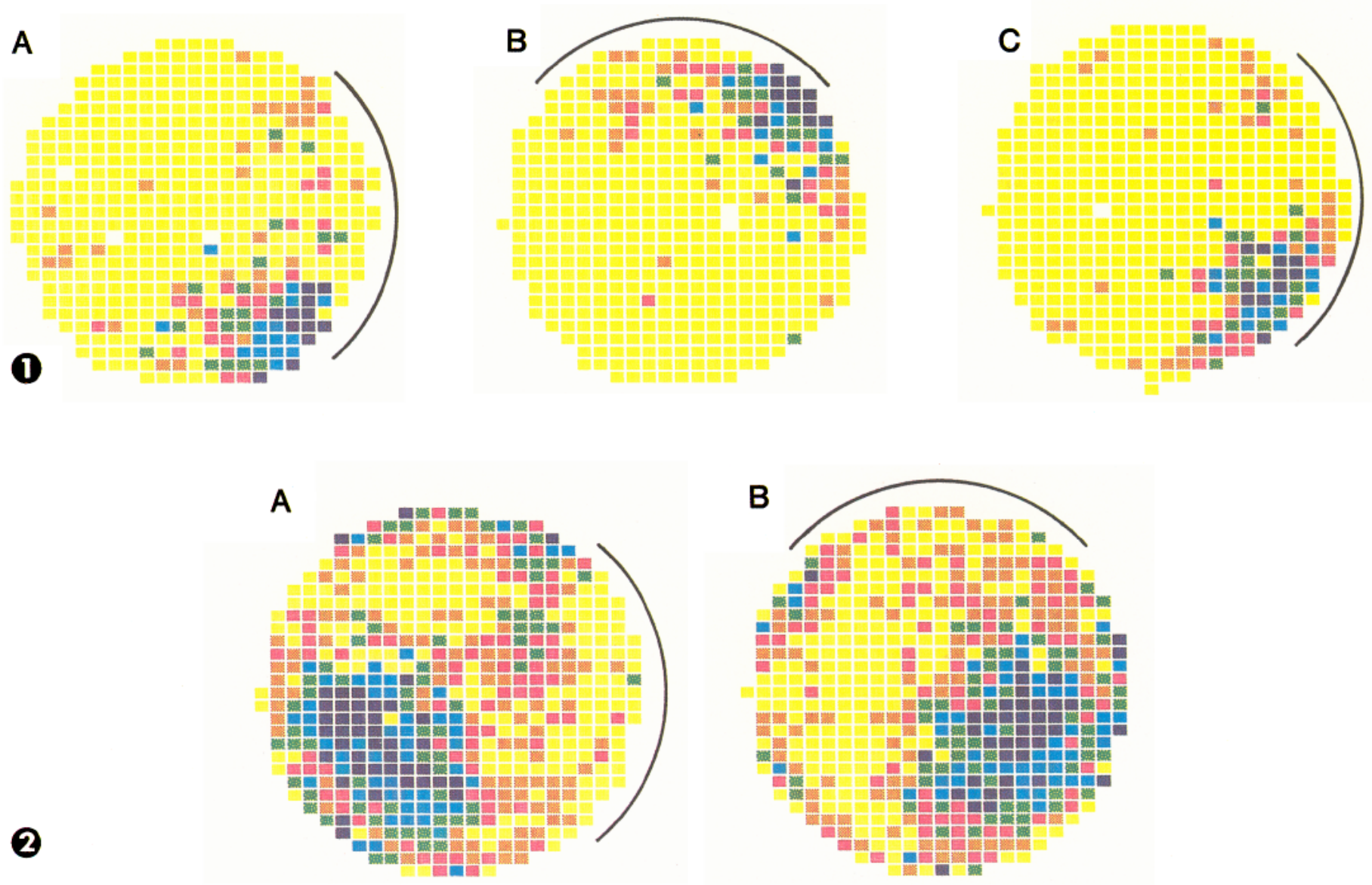

Figure 1. Effects of rotating the cue card plus walls on the angular position of a firing field. The rate map in $A$ was obtained with the cue card in its standard position (centered at 3 o'clock, as indicated by the line to the right of the map). A firing field is visible at about 5 o'clock. When the session was over, the rat was removed from the apparatus and the walls, with the card attached, were rotated $90^{\circ}$ counterclockwise. A second session, whose outcome is summarized in $B$, was then run. It is clear that the firing field rotation was virtually the same as the wall rotation. A third session was then run in which the walls were returned to their original position. In $C$, it is seen that the firing field also went back to its original position. Cell R3U7; CA3/4. A, Session R3S45B8. A session name consists of the rat number (R3), the session number for the rat (S45), and the duration of the session in minutes (B8). Median firing rates for colors: yellow $=0.0$; orange $=0.78$; red $=2.45$; green $=6.00$; blue $=11.43$; purple $=18.46$. Since the color order for increasing firing rates is the same for all maps in this paper, only the sequence of median firing rates will be stated for the rest of the maps. $B$, Session R3S46B8. Median firing rates: $0.0 ; 0.87 ; 6.00 ; 9.23 ; 21.82$. $C$, Session R3S47B8. Median firing rates: $0.0 ; 1.00 ; 3.36 ; 10.00 ; 19.59$.

Figure 2. Effects of rotating only the cue card on the angular position of a firing field. $A$ is a firing rate map from a standard session in the small cylinder. When this session was finished, the rat was put back in its home cage. The cue card was then detached from the cylinder wall and reattached $90^{\circ}$ counterclockwise from its usual position (i.e., at 12 o'clock). The session whose rate map is shown in $B$ was then run. Although the main field is rather large and there are other regions of relatively intense activity, it is clear that the overall spatial pattern rotated by approximately $90^{\circ}$. R54U1; CA3/4. A, R54S1B16. Median firing rates: $0.0 ; 0.72 ; 1.78 ; 3.87 ; 6.90 ; 12.17$. B, R54S3B16. Median firing rates: 0.0; 0.69; $1.62 ; 3.09 ; 5.39$; 9.93 .

card was rotated, by detaching it and reattaching it at 9 o'clock. This manipulation also resulted in an equal rotation of the firing field (Fig. $2 B$ ). The same effect was seen in each of 8 repeats of this experiment. Thus, the cue card exerts stimulus control over the angular position of the firing field, although the control is not necessarily exclusive to the card.

To test the possibility that firing fields follow the position of a local detail of the cue card, 7 cells were recorded in the small cylinder and in a copy of the cylinder that contained a different card; twice, the different card was an exact copy, and 5 times it was a tan formica sheet of the same dimensions. In each case, the firing field appeared the same in both cylinders. In each of 2 cases, rotation of the second small cylinder produced equal firing field rotation. Together, these results imply that details of the cue card are not used for anchoring the angular coordinate of place cell firing, and that stimulus control resides in the overall appearance of the card.

Accuracy of field rotation after card rotation. To numerically estimate the amount of firing field rotation caused by cue-card rotation, the firing rate in each $6^{\circ}$ wedge of the cylinder is found for the standard (card at 3 o'clock) and rotated sessions. The correlation coefficient between the 2 angular firing rate distributions is then calculated. Next, the distribution from the standard session is shifted counterclockwise by $6^{\circ}$ and a second correlation coefficient is found. The distribution from the standard session is then shifted in $6^{\circ}$ steps and the correlation is recalculated until 60 steps are taken and the entire circle is complete. The set of correlation values obtained in this way will be referred to as the "angular cross-correlation." The angle at which the maximum correlation occurs will be referred to as 
$A_{\max }$, and is an estimate of the amount of field rotation causcd by cue-card rotation. In Figure $3 A$, the angular cross-correlation is given for the sessions of Figure $1, A, B ; A_{\max }$ was at $96^{\circ}$. Figure $3 B$ gives the angular cross-correlation for the sessions in Figure $2, A, B$; the $A_{\max }$ was again at $96^{\circ}$.

To estimate the precision of field rotation, the difference $(\Delta R)$ is taken between the card rotation $\left(R_{\mathrm{c}}\right)$ and the field rotation $\left(R_{\mathrm{f}}\right)$, as measured by $A_{\max }$. The mean $\Delta R$ for 15 rotation experiments was $3.8^{\circ} \pm 14.1^{\circ} \mathrm{SD}$. These values may be compared with those obtained from pairs of standard sessions. For 20 pairs of standard sessions, $R$ was $0^{\circ}$, with a standard deviation of $4.35^{\circ}$. A $t$ test for differences between the mean errors showed that they were the same within experimental error $(t=1.20$; $p<0.12$ ). By contrast, an $F$ test showed that the variance of $\Delta R$ for rotations was greater than the variance for replications $(F=10.5 ; p<0.01)$. Thus, the angular position of the field was more variable after rotations than after replications. This suggests that features of the environment besides the cue card may act as partial determinants of the angular coordinate of fields.

Effect of reducing the size of the cue card. Additional information about the stimulus control exerted by the cue card over place cell firing can be gained by changing the size of the card. Figure $4 A 1$ is a map from a standard session with a $100^{\circ}$ cue

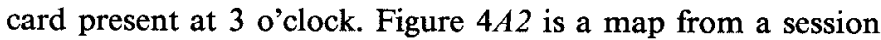
using a half-width $\left(50^{\circ}\right)$ card also centered at 3 o'clock. The spatial firing pattern was substantially the same in the 2 sessions. The spatial firing pattern was basically unchanged for all 10 cells recorded when a half-width cue card was substituted for the full card. In some cases, it appeared that using the half-width card led to a rotation of the firing field; an example is given in Figure $4, B 1$ and $B 2$. This observation opens up the possibility of identifying the part of the card that acts to establish the reference angle by measuring the extent of the rotation associated with different width replacements.

As noted, $A_{\max }$ measures the amount that a firing field has rotated between 2 sessions. By contrast, $r_{\max }$, the magnitude of the correlation at $A_{\max }$, measures the similarity of 2 angular firing distributions. The average $r_{\max }$ for pairs of standard sessions run on the same cell was 0.876 (Table 1). The average $r_{\max }$ from standard session and half-card session pairs was 0.888 . Since these are indistinguishable $(t=0.314 ; p<0.38)$, it appears that halving the card width did not disrupt angular firing distributions. The mean $r_{\max }$ for standard/rotation pairs was 0.837 . A $t$ test revealed that the mean $r_{\max }$ for standard/rotation pairs was somewhat lower than for standard/standard pairs $(t=1.30 ; p \approx$ $0.1)$. By contrast, another manipulation (card removal) leads to a clear reduction of the mean $r_{\max }$ (see Tablc 1 and bclow).

Effects of removing the cue card. From the work of O'Keefe and Conway (1978) on the effects of deleting from 1 to all 4 of a set of salient stimuli, it might be expected that removing the cue card would lead to a drastic disruption of the spatial firing pattern. In a minority of cases, this was the result. A second possible effect of card removal would be for the firing pattern to become annular; it might still be possible for the cell to fire reliably as a function of distance from the center of the apparatus even if angular specificity disappeared. Other possibilities include a cessation of firing or a loss of spatial cohesiveness, with a similar firing rate over the area of the apparatus.

Contrary to the possibilities given above, card deletion usually produced only a rotation of the field to an unpredictable angular position. In other words, the spatial firing distribution most often appeared unchanged with regard to radial position, shape,
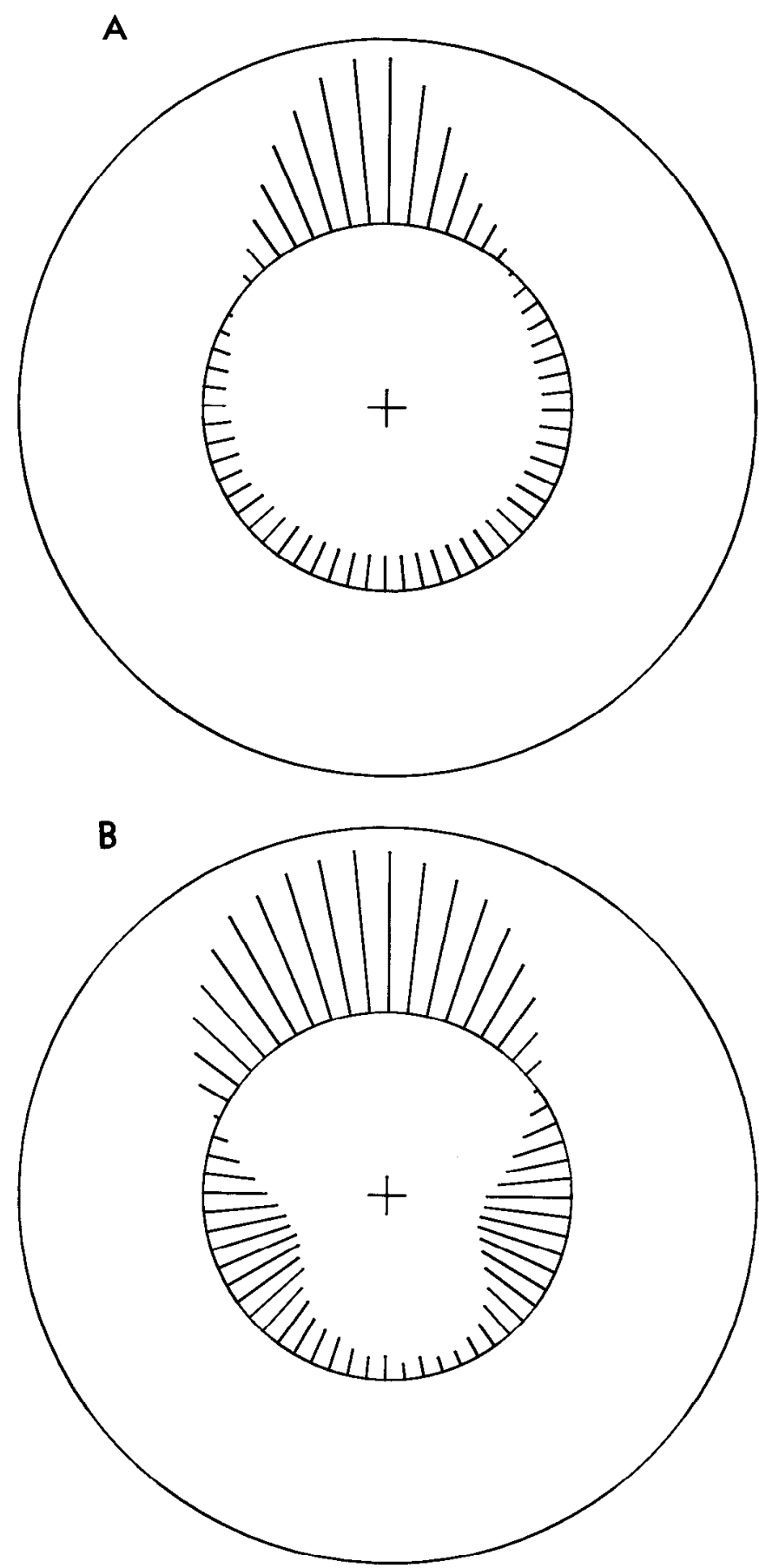

Figure 3. Examples of the rotational cross-correlation of pairs of angular firing rate distributions. $A$, for the maps of Figure $1, A, B ; B$, for the maps of Figure $2, A, B$. The small circle represents a correlation coefficient of 0.0 , the large circle represents a correlation coefficient of 1.0 , and the cross at the origin represents a correlation coefficient of -1.0 . Zero degrees is on the right, corresponding to the standard cue card position at $30^{\prime}$ clock. The length of the vector at $0^{\circ}$ is the correlation coefficient with the cylinder in its original position relative to the laboratory for both sessions. As expected from the original maps, the maximum correlation between the standard and rotated angular rate distributions $\left(R_{\max }\right)$ occurs near $90^{\circ}$. In $A, A_{\max }=96^{\circ}$ and $R_{\max }=0.89$. In $B, A_{\max }=96^{\circ}$ and $R_{\max }=0.88$. Note the interesting pattern of correlation values near $270^{\circ}$ in $B$, where the correlation coefficient reaches a local maximum. The reason for this effect is visible in the original rate maps (Fig. 2, $A, B$ ), which both show a secondary area of relatively high firing diametrically opposite the main firing field. 
A 1

Figure 4. Halving the width of the cue card does not disrupt spatial firing patterns. $A$, Rate map of $A l$ is from a standard session. For the session in $A 2$, a cue card that occupied $50^{\circ}$ instead of $100^{\circ}$ of arc centered at 3 o'clock was used. The strong similarity between the firing fields in $A 1$ and $A 2$ indicates that changing the width of the card had little effect on the activity of this cell. The rotational cross-correlation reached a maximum of 0.923 with $A_{\max }=12^{\circ}$. A second example of the effects of a halfwidth cue card is shown in $B$. The rate map in $B 1$ is from a standard session. For the map in $B 2$, a $50^{\circ}$ cue card centered at 3 o'clock was used. Note that the field appears the same except for a rotation of about $30^{\circ}$ clockwise. $A$, R30U7; CA3/4. B, R37U4; CA1. A1, R30S19B16. Median firing rates: 0.0 ; $0.82 ; 2.50 ; 4.86 ; 6.67 ; 9.23$. $A 2$, R30S20B16. Median firing rates: 0.0 ; $0.71 ; 2.67 ; 4.62 ; 7.83 ; 12.00$. B1, R37S3B16. Median firing rates: 0.0 ; $0.47 ; 3.00 ; 8.44 ; 17.73 ; 26.27$. B2, R37S4B16. Median firing rates: 0.0 ; $0.83 ; 2.19 ; 4.62 ; 8.33 ; 16.91$.
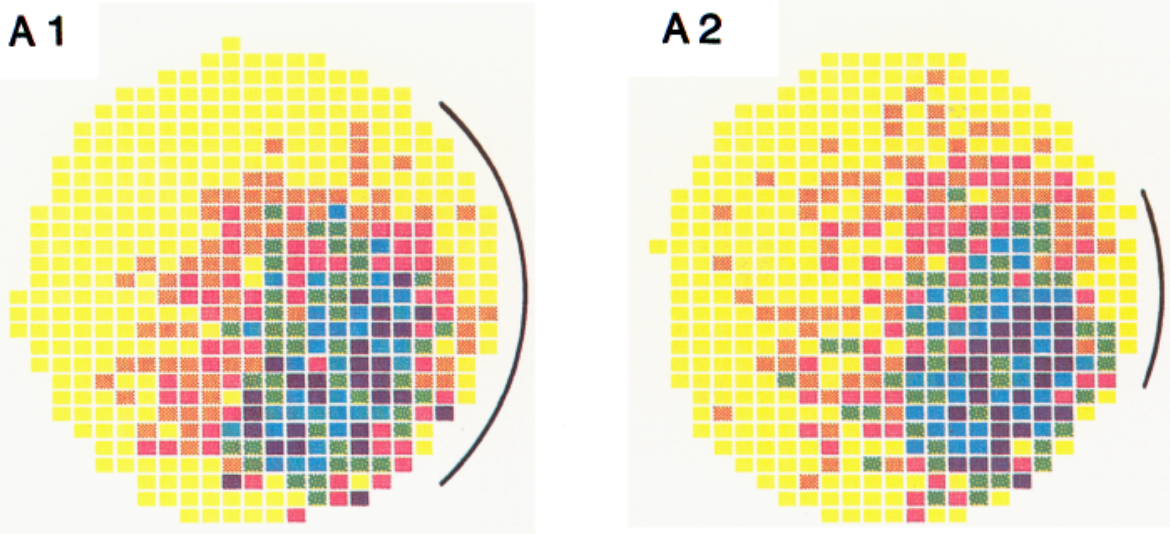

B 1

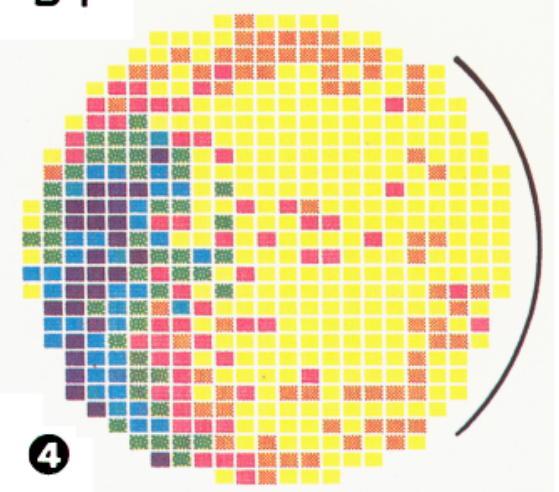

B2

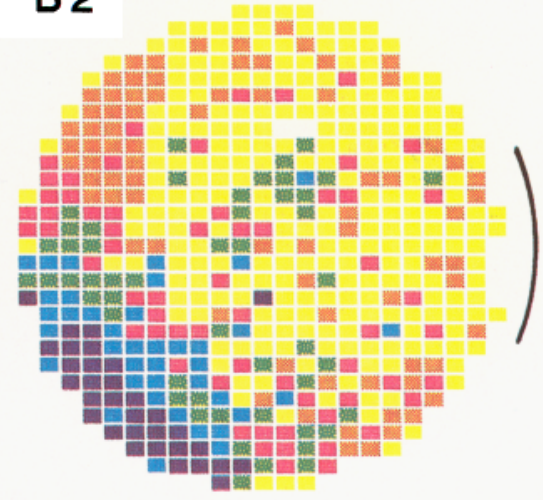

size, and intensity, and differed from the standard session only in angular coordinate. Three deletion experiments are illustrated in Figure 5. Figure 5, $A$, and $B$, shows a session with no cue card bracketed by standard sessions, whereas Figure $5 C$ shows a more complicated sequence of sessions fully described in the legend. It is our impression from the maps of Figure 5 that the crispness of the spatial firing pattern is reduced in no-card sessions. This impression is supported by the data in Table 1 , which show that the $r_{\max }$ between standard sessions and no-card sessions is lower than $r_{\max }$ for the simpler manipulations mentioned above. (The tabulated value is an average for 11 of 12 repeats of the cardremoval experiment; the other cell will be considered below, since its firing pattern was irreversibly changed by the card removal.) We conclude that cue deletion produces a measurable loss of spatial coherence, but that fields are fundamentally unchanged by this procedure.

The main implication of this finding is that the card is sufficient, but not necessary, to anchor the angular coordinate. The persistence of reliable, nonannular, location-specific firing after card removal means that the angular coordinate of firing can be associated with a variety of stimuli, and that the angular location of the field can vary independently of other firing field properties. The necessary information may be provided by other constant stimuli (e.g., the seam of the cylinder that is usually covered by the card), marks that the animal creates itself (e.g., urine spots), or dead reckoning, but some other way of obtaining a stable coordinate system must exist. Examples illustrating this are contained in the maps of Figure 5 . For the first cell illustrated in Figure 5, the field, in the absence of the cue card (5A2), went to a position different from that seen with any of the card rotations (including the standard position) tried with this cell. By contrast, for the second cell, the field with no cue card (5B2) is where it would be expected to be if the card were present in its standard position. The field went to this position despite the fact that the session immediately before the one in Figure $5 B 2$ was done with the cue card rotated by $180^{\circ}$ (not shown). In the absence of the cue card, the firing of this cell apparently was tied to stimuli associated with the standard orientation of the apparatus. The sequence for the cell in Figure $5 C$ shows another effect of card removal. Figure $5 C 1$ shows a standard session. Figure $5 C 2$ shows the outcome of rotating the card $90^{\circ}$ counterclockwise. When the card was removed for the next session (Fig. 5C3), the field stayed in the $90^{\circ}$ rotated position. Next, another standard session was run (Fig. 5C4), with the expected result. A second card-removal session (Fig. 5C5) followed immediately afterwards. This time, the field was found in the position appropriate for the standard card placement. It therefore appears that the firing of this cell reflected information retained about the previous position of the card in the laboratory frame.

Summary of cue card experiments. The data presented show that place cell activity is controlled in part by the cue card. They further imply that the cue card is properly described as a polarizing stimulus, since its control is almost completely limited to fixing the angular coordinate of firing with respect to the laboratory frame. The fact that the card can be removed without affecting the shape, size, or radial position of firing fields will be considered further in the Discussion.

\section{Manipulations of the apparatus walls}

In this series of experiments, we showed what happens to place cell firing when the small cylinder is transformed in shape to produce several other apparatuses. It was shown above that 
Table 1. Peak rotational correlation coefficients for replications, rotations, half-card, and card-removal experiments ${ }^{a}$

\begin{tabular}{lllll} 
& $\begin{array}{l}\text { Replica- } \\
\text { tions }\end{array}$ & Rotations & Half-card & $\begin{array}{l}\text { Card- } \\
\text { removal }\end{array}$ \\
\hline$N$ & 20 & 15 & 6 & 11 \\
Mean $r_{\max }$ & 0.884 & 0.837 & 0.886 & $0.680^{b}$ \\
Mean $Z_{\max }$ & 1.393 & 1.211 & 1.414 & $0.829^{b}$ \\
$Z_{\max } S D$ & 0.432 & 0.397 & 0.344 & 0.399 \\
\hline
\end{tabular}

a The values of interest are the peak values of the rotational cross-correlation between pairs of sessions. For statistical comparisons, the correlation coefficient $\left(r_{\text {max }}\right)$ for each pair of sessions is first transformed into a " $z$ score"; means, standard deviations and $t$ tests are performed on the transformed values. To obtain the statistics in terms of $r_{\max }$, the inverse transformation is done. (See Walker and Lev, 1953.)

${ }^{b}$ The mean value of $r_{\max }$ for card removals is significantly lower $(p<0.01)$ than the values of $r_{\max }$ for any of the other manipulations. We take this to indicate that there is a detectable degradation of field structure when the cue card is removed.

swapping the cylinder for a copy had no effect on place cell firing. It follows that any effects seen with nontrivial transformations cannot be attributed to changes in incidental details of the animal's environment. The first transformation was equal scaling in all dimensions; this yielded the large cylinder, with diameter and height twice that of the small cylinder. Second, topological transformations were done to produce rectangular apparatuses.

To classify the responses of place cells to these manipulations, a cell is recorded in 2 apparatuses and the resulting firing rate maps are inspected. The cell is assigned to 1 of 3 categories, depending on whether it has a field in neither, one, or both of the apparatuses. A cell is considered to have a field in an apparatus if there is a region at least 9 pixels in area such that the firing rate in each pixel is greater than $1.0 \mathrm{AP} / \mathrm{sec}$ (Muller et al., 1987). The 3 classes of units are called N/N (no-field/no-field), F/N (field/no-field), and F/F (field/field) cells. F/F units are further split into 2 types, which will be referred to as UF and RF cells. A cell is UF if its field in one apparatus appears to be $u$ nrelated to its field in the other. By contrast, the fields of RF cells in the 2 apparatuses must bear a simple relationship to each other.

Effects of scaling on location-specific firing. Two sets of scaling experiments were run, one with cylinders and the other with rectangles. We first consider cells that were recorded in both the small and large cylinders ( $n=25$; see Table 2$)$. Place cells that had a firing field in neither cylinder ( $3 \mathrm{~N} / \mathrm{N}$ units) or in only one ( $9 \mathrm{~F} / \mathrm{N}$ units) cylinder do not require illustration, since the observed firing patterns were described in the previous paper (Muller et al., 1987). The remaining 13 (F/F) cells had firing fields in each cylinder.

Nine of the F/F cells were considered RF cells because they had fields whose positions were similar (in the strict geometrical sense) in the 2 cylinders. In other words, the relationship between the fields mimics the change in the structure of the apparatus. The field-scaling phenomenon is documented in Figure $6, A-C$. The 4 remaining $\mathrm{F} / \mathrm{F}$ cells were considered UF cells, since an arbitrary transformation rule would have been needed for each cell. An example of a UF cell is shown in Figure $6 D$.

The angular cross-correlation method can be used to show that RF cells form a class. The rotation that produces the maximal correlation between the angular firing rate distributions $\left(A_{\mathrm{max}}\right)$ in the 2 cylinders is found for each $\mathrm{F} / \mathrm{F}$ cell. The binomial distribution can then be used to calculate the probability of finding a certain number of cells with $A_{\max }$ in a given range of
Table 2. Occurrence of the different cell types for apparatus pairs in the 4-apparatus experiment

\begin{tabular}{llclll} 
Apparatus pair & N/N & N/F & RF & UF & Total $^{a}$ \\
\hline Lg. cyl./sm. cyl. & 3 & 9 & 9 & 4 & 25 \\
Lg. rect./sm. rect. & 6 & 5 & 7 & 1 & 19 \\
& & & & & \\
Sm. cyl./sm. rect. & $0^{b}$ & 12 & 1 & 9 & 22 \\
Lg. cyl./lg. rect. & 5 & 8 & 0 & 5 & 18 \\
Sm. cyl./lg. rect. & 1 & 12 & 0 & 5 & 18 \\
Lg. cyl./sm. rect. & 3 & 10 & 0 & 7 & 20
\end{tabular}

a total of 18 cells were run in all 4 apparatuses; the larger numbers for some apparatus pairs reflect the outcomes of experiments in which the cell was lost before the whole experiment was done and a few experiments that were intentionally less complete.

${ }^{b}$ Since cells were selected for study only if they had a field in either the small cylinder or the small rectangle, this number must be 0 .

angles, using the angular rate distribution in the small cylinder as the reference. For example, the probability for a single cell that independent fields in the 2 cylinders will have their $A_{\max }$ in the range $\pm 30^{\circ}$ is one-sixth. Of $13 \mathrm{~F} / \mathrm{F}$ cells, 9 cells, all of which were RF, satisfied this criterion. The binomial probability of 9 or more successes in 13 trials with $p=1 / 6$ is $\ll 0.001$. The low probability is not a result of the arbitrary choice of $\pm 30^{\circ}$ resolution. If the acceptable range is $\pm 15^{\circ}$ or $\pm 7.5^{\circ}$, in each case 5 of $13 \mathrm{~F} / \mathrm{F}$ cells meet the criteria. The probability of 5 successes in 13 trials is 0.0029 for $\pm 15^{\circ}$ and $\ll 0.001$ for $\pm 7.5^{\circ}$. Thus, the notion that fields scale in scaled cylinders is supported by a simple statistical test.

The results with cylinders are confirmed by scaling experiments in rectangular apparatuses on a total of 19 place cells. Figure 7, $A, B$, contains colored maps for 2 of the $7 \mathrm{RF}$ cells; Figure $7 C$ contains maps for 1 of the 2 UF cells. Thus, field scaling is not peculiar to cylinders. Table 2 shows that the fractions of place cells that fall into the field pair classes are substantially the same for rectangles as for cylinders.

If scaling were ideal, fields in large apparatuses should have 4 times the area of those in the small versions. In reality, the maximum ratio of field size in the cylinders for an individual RF cell was 2.06, and the minimum 0.24; the mean field size ratio was 0.87 . Part of this discrepancy is probably a sampling error due to the smaller amount of time that the rats spend per unit area in the large cylinder. To test this possibility, 2 calculations were done. In the first, the average time spent per unit area was made equal by considering only the first $4 \mathrm{~min}$ of small cylinder sessions. This raised the mean ratio of field size of the large to the small cylinder from 0.87 to 1.73 . In the sccond calculation, rectangular sets of 4 pixels were collapsed into larger pixels for the large cylinder; this made the mean dwell time per pixel the same for sessions of equal duration. In this case, the mean size ratio was 2.49 . We conclude that sampling problems may be obscuring an interesting relationship between field sizes in the large and small cylinders, and that further work in this direction is warranted.

A topological transformation: the 4-apparatus experiment. Up to now, rectangular apparatuses have been used to demonstrate that the place cell classes are not peculiar to cylinders, and that field scaling takes place in apparatuses of other shapes. In this section, the firing field transformations seen when a cell is recorded in both cylinders and rectangles are described. Recordings were made from 18 individual cells in each of the 4 primary apparatuses (the large and small cylinders and the 2 rectangles) 
A 1
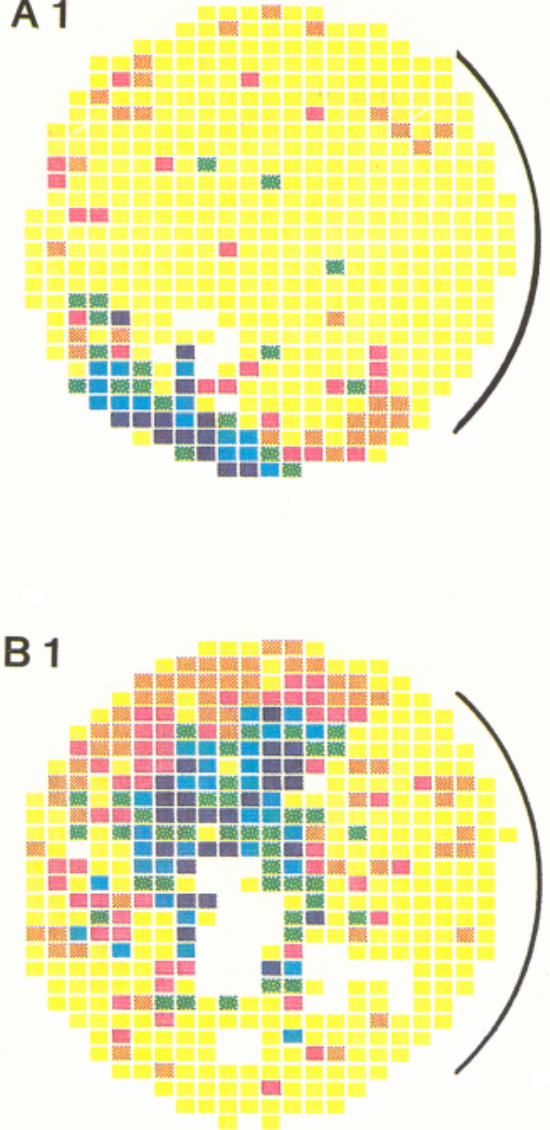

C 1

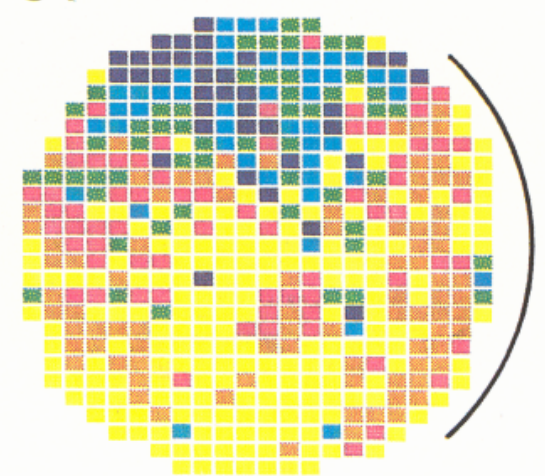

A 2

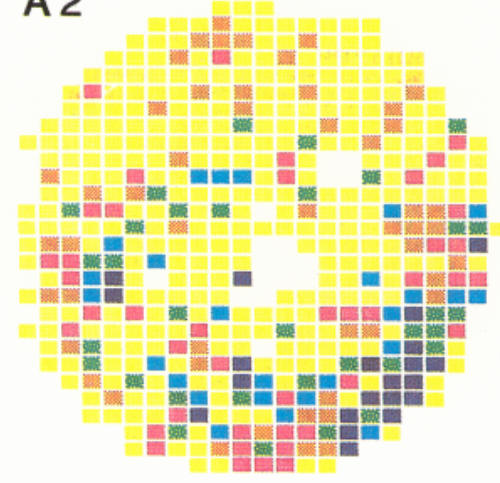

B 2

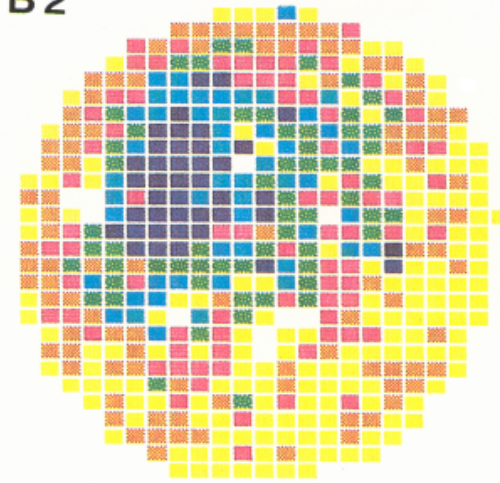

C2

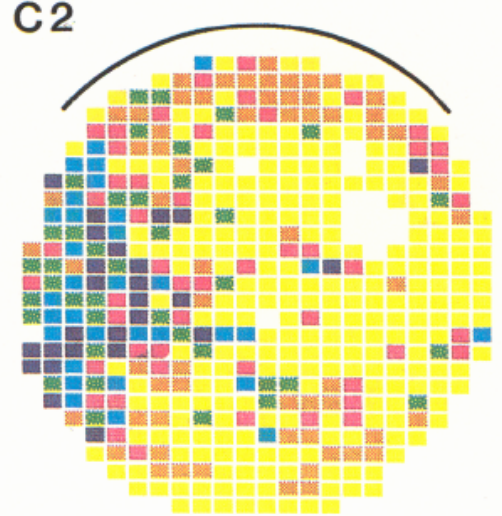

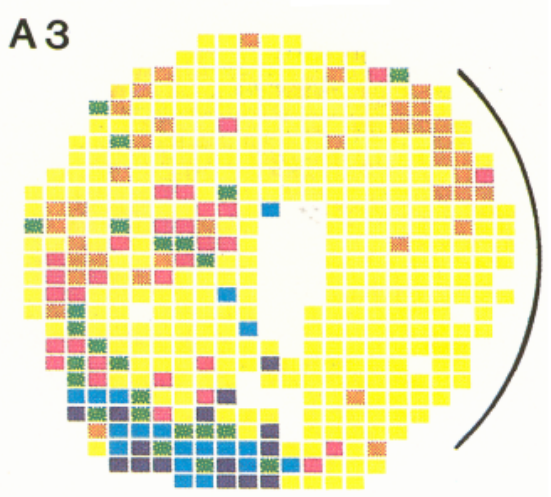

B 3

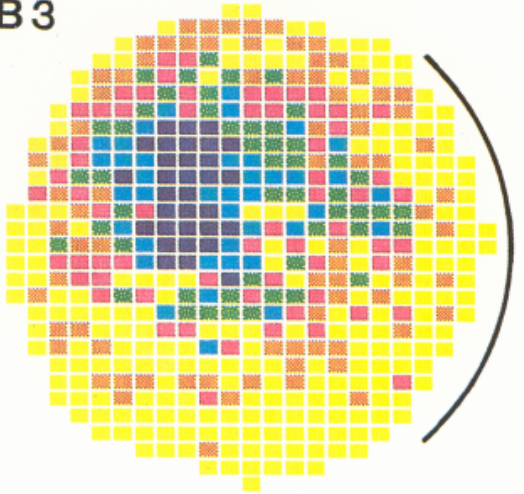

C 3

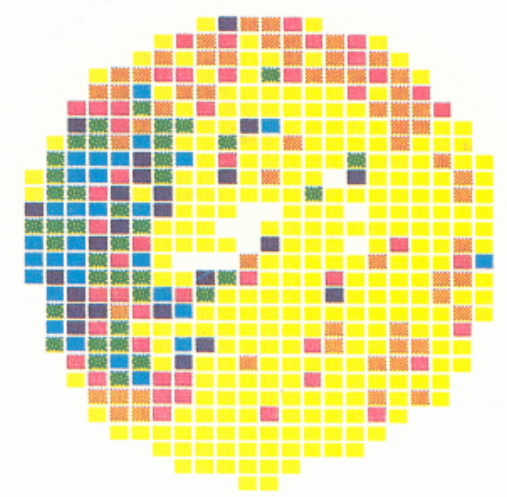

5
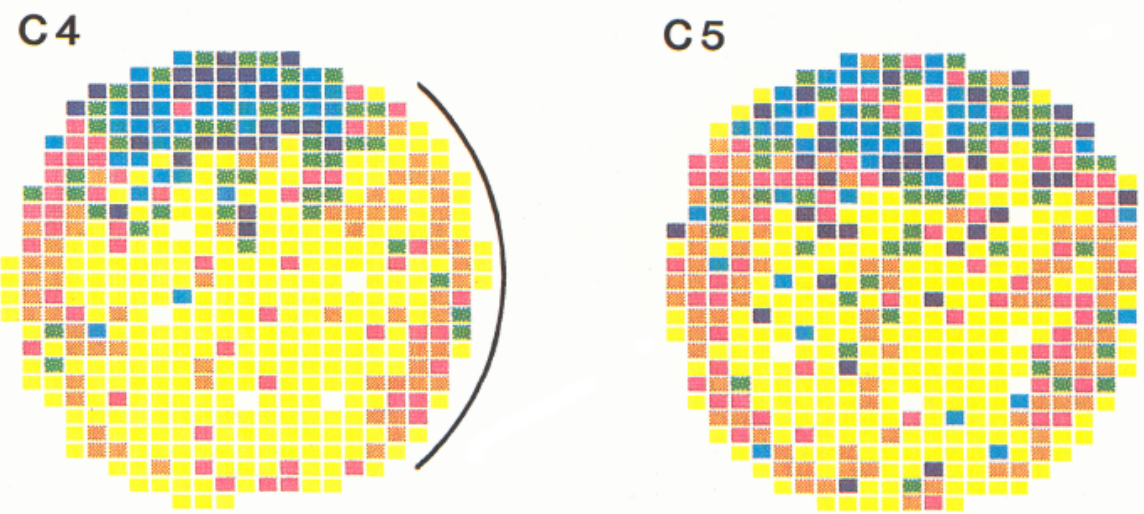
and from several other cells in 2 or 3 of the apparatuses. Table 2 summarizes the number of cells that fell into the 4 categories defined above for each of the 6 apparatus pairs. The important point in Table 2 is the contrast in cell type distribution between the cylinder-rectangle apparatus pairs and the isotropically scaled apparatus pairs. In particular, the RF cells are missing in the 4 cylinder-rectangle pairs. This means that we saw no consistent connections between field pairs in cylinders and rectangles, and, in particular, that firing fields did not transform topologically along with the apparatus shape. Instead, it is impossible to predict the nature of a firing field in a cylinder from a knowledge of the cell's field in a rectangle, and vice versa.

\section{Introducing barriers into the apparatus}

For the 2 classes of manipulations presented so far (cue card and apparatus shape), the firing field of every cell is changed, albeit in very different ways. The effects of putting a barrier into the otherwise free space of the small cylinder differ in that only those cells whose fields arc in the vicinity of the barrier are strongly affected. The basic barrier effect is shown in Figure 8. Figure $8 A$ shows the firing pattern for a standard session in which no barrier was present. The rate map for this session was used to locate the cell's firing field, so that before the next session a $23 \times 23 \mathrm{~cm}$ opaque (fiberboard) sheet could be vertically set to cut the field in half. Figure $8 B 1$ shows that the barrier reduced the field size. The strength of the barrier's effect is masked, however, by our practice of autoscaling the firing rates in the color categories (Muller et al., 1987). The true magnitude of the firing decrement is seen in Figure $8 B 2$, which is another rate map for the same barrier session in which the firing rate range for each color category is the same as in the standard session map (Fig. 8A). Similar reductions in firing rate were seen for 9 of 10 cells when a barrier was placed to bisect the erstwhile field; the exception will be discussed below. The maps in Figure $8 C 1$ (autoscaled) and $8 C 2$ (scaled as in Fig. $8 \mathrm{~A}$ ) are for a second standard session. They show that field size and intensity returned to their original values after the barrier was removed. In all subsequent barrier experiment figures, the rate maps for a cell use color-coded firing rate categories obtained from autoscaling the rates for an initial standard session.

The sequence of sessions in Figure 9 demonstrates that the degree to which the barrier encroachcs on the firing field determines the magnitude of its effect. In Figure 9, $A$ and $B$, are maps for a replication of the experiment in Figure 8. In Figure
$9 C$, the barrier extended from the cylinder wall inwards along the radius from 12 o'clock. The field is mainly unchanged by this manipulation, although it is somewhat smaller than in the standard session. (The diminution of the background firing in the right half of Fig. 9, $C$ and $D$, is a second-order effect compared to the changes in firing in the neighborhood of the field, and will not be considered further.) After another standard session (not shown), the barrier was placed tangentially to the part of the field nearest the center of the cylinder (Fig. 9D). In response, the field contracted away from the barrier, although the field intensity was not reduced. Great attenuation of the field was seen again when the barrier was in the same position as in Figure $9 B$ (not shown). Moving the barrier centrally, so that its outer end was away from the wall (Fig. 9E) also produced a strong attenuation of the field. The standard session in Figure $9 F$ shows that the field was less crisp at the end of the series of seven 16 min sessions. In another series of sessions (nol shown), it was seen that bisecting a centrally located field with a barrier from 3 to 9 o'clock was just as efficient in reducing the cell's firing as bisecting the field with the barrier from 6 to 12 o'clock.

We conclude from the differential effects of barrier location that alterations in place cell activity are mainly confined to cells whose fields lie near the barrier, or, in other words, that the control exerted by the barrier is local in nature. The same experiments indicate that the power of the barrier to alter a firing field does not come from the change it produces in the visual appearance of the apparatus from the perspective of that field, since putting the barrier between the field and the rest of the apparatus was much less effective than placing the barrier in the field. This supposition is bolstered by the experiments shown in Figure 10. Figure 10, $A-C$, again shows the effects of putting the opaque barrier through the field and then removing it. Figure $10 D$ is the rate map oblained when a transparent (lucite) barrier, instead of the opaque barrier, was used to bisect the field. The effectiveness of the transparent barrier renders implausible any simple visual explanation of the barrier phenomenon. In the experiment of Figure $10 E$, the lead base that ordinarily supported the barriers was used by itself to bisect the cell's firing field. The weak effect suggests that merely putting a stimulus into the field is not sufficient to disrupt location-specific firing. Instead, it appears that the basic effect depends on the "barrierness" of the object placed in the field, and not on the details of its stimulus properties.

There is, however, a problem if all cells with fields close to

\section{$\leftarrow$}

Figure 5. Effects of removing the cue card on place cell firing. Map in $A 1$ is from a standard session run in the small cylinder. The cue card was removed from the apparatus in the session shown in $A 2$; the spatial firing pattern is substantially the same, but the angular position of the firing field is in a completely different location. The cell recorded in $A$ was the first one recorded from this animal, and the cue card had never been put in a position that would lead to an expectation of finding the field near 4:30. In other words, the field rotated to a position that was unpredictable from any past experience of the animal. $A 3$ shows the map from a second standard; the ficld is back in its "proper" position (this session was done on the day after those in $A 1$ and $A 2$ ). The sequence of sessions shown in $B$ is the same as in $A$; standard, no-card, standard. In this case, however, the position of the field in the absence of the cue card was appropriate for the card's being in the standard position. This was true even though another session (not shown) was done between those in $B I$ and $B 2$, in which the cue card was attached at $90^{\circ}$ clock (180 away from the standard position). The sequence of sessions in $C$ also shows maintained spatial firing in the absence of the cue card (C3 and $C 5)$. For this cell, however, the angular position of the firing field in the first no-card session $(C 3)$ was the same as in the preceding session $(C 2)$, with the card rotated $90^{\circ}$ counterclockwise. Similarly, the angular position of the field in the second no-card session (C5) was the same as it was in the preceding standard session $(C 4)$. This cell apparently was able to "remember" the last position of the cylinder during no-card sessions. $A$, R8U1; CA1. B, R14U1; location not verified. $C$, R18U1; CA1. AI, R8S3B8. Median firing rates: $0.0 ; 0.62 ; 2.00 ; 4.62 ; 10.69 ; 18.16 . A 2$, R8S4B8. Median firing rates: 0.0; $0.82 ; 2.08 ; 3.53 ; 5.29 ; 9.00$. A3, R8S5B8. Median firing rates: $0.0 ; 0.80 ; 2.86 ; 4.80 ; 10.23 ; 20.87$. B1, R14S1B16. Median firing rates: $0.0 ; 0.81$; $2.88 ; 5.98 ; 11.67 ; 21.43 . B 2, \mathrm{R} 14 \mathrm{~S} 3 \mathrm{~B} 16$. Median firing rates: $0.0 ; 1.68 ; 4.29 ; 8.57 ; 15.65 ; 26.67 . B 3$, R14S5B16. Median firing rates: $0.0 ; 0.92$; $2.77 ; 5.71 ; 9.47 ; 21.60 . C 1, \mathrm{R} 18 \mathrm{~S} 1 \mathrm{~B} 16$. Median firing rates: $0.0 ; 0.86 ; 1.82 ; 3.40 ; 5.71 ; 9.43 . C 2$, R18S3B16. Median firing rates: $0.0 ; 0.97 ; 2.43$; $4.00 ; 6.67 ; 10.00$. C3, R18S4B8. Median firing rates: $0.0 ; 1.11 ; 2.86 ; 4.92 ; 6.92 ; 10.50$. C4, R18S5B8. Median firing rates: $0.0 ; 0.97 ; 2.32 ; 5.00 ;$ $8.57 ; 12.73$. C5, R18S6B8. Median firing rates: $0.0 ; 1.22 ; 2.93 ; 4.39 ; 6.32 ; 10.29$. 


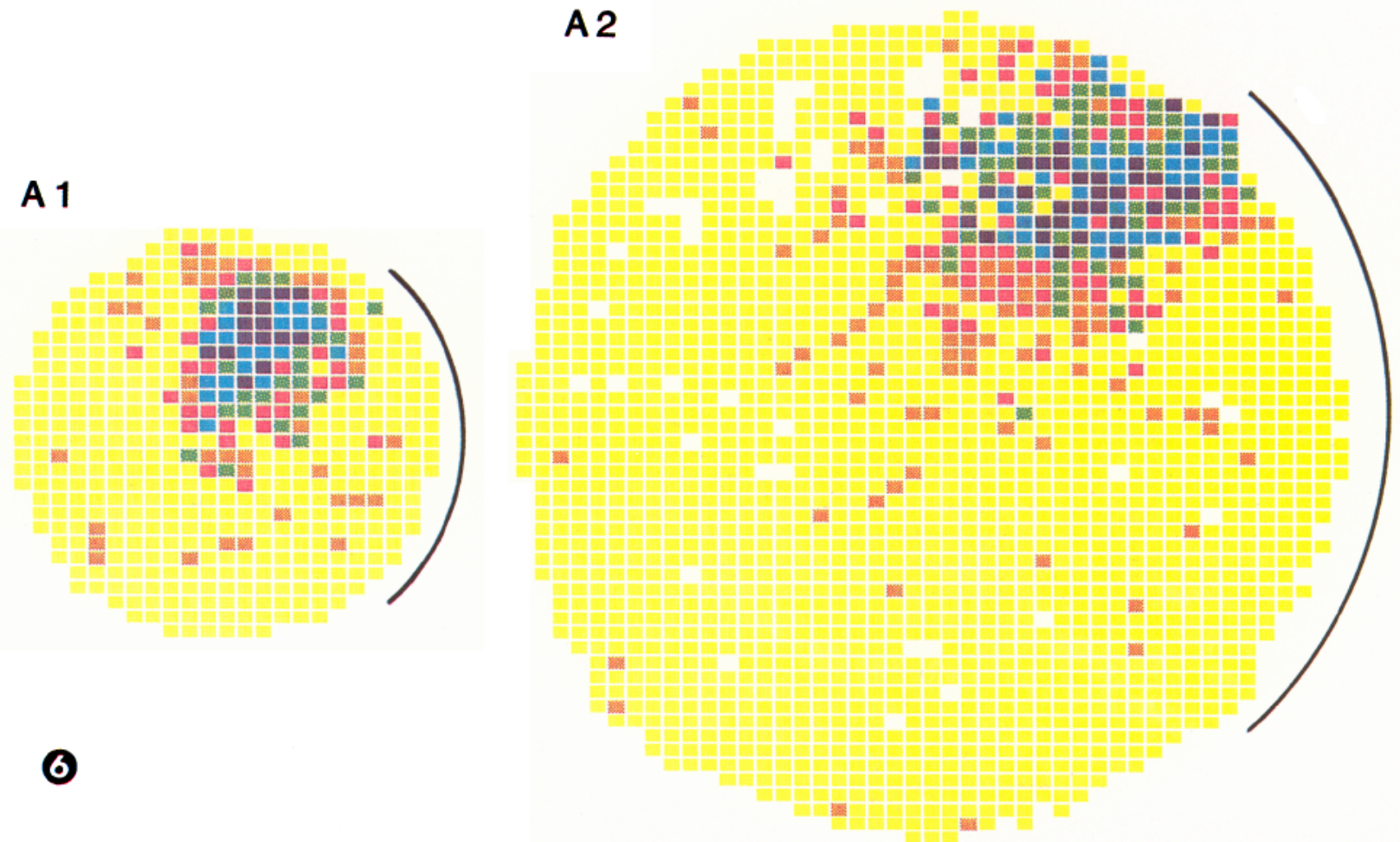

Figure 6. The response of 4 place cells to isotropic scaling of the small cylinder. $A, B$, and $C$ are examples of units that we classified as RF cells; in each case, the relative position of the firing field in the large cylinder is the same as that in the small cylinder. $C$, Both fields in the small cylinder have scaled counterparts in the large cylinder. The small firing patch at 7 o'clock in $B$ is due to imperfect discrimination from a second cell on the same wire as the target cell. $D$, An example of a UF cell; the fields in the 2 cylinders are not obviously related to each other. It is true that a rotation plus scaling might transform the firing pattern in the small cylinder into a reasonable replica of the firing pattern in the large cylinder, but the use of a rotation is arbitrary, since the white cue card was in the same relative position in both apparatuses. In $B, C$, and $D$, the rate map of the large cylinder has been reduced 4:1. $A$, R3U4; CA 1. B, R7U1C; CA1. C, R29U1; CA3/4. D, R3U6; CA3/4. Note that 1 of the RF cells and the UF cell were recorded from the same rat. $A 1$, R3S20B16. Median firing rates: $0.0 ; 0.45 ; 1.73 ; 3.90 ; 8.57 ; 18.46$. $A 2$, R3S22B16. Median firing rates: $0.0 ; 2.14 ; 7.14 ; 13.33 ; 19.41 ; 32.00$. B1, R7S9B8. Median firing rates: $0.0 ; 1.12 ; 3.02 ; 6.90 ; 12.35 ; 21.11$. B2, R7S11B16. Median firing rates: 0.0 ; $2.58 ; 6.00 ; 11.43 ; 20.00 ; 35.46$. $C 1$, R29S1B16. Median firing rates: $0.0 ; 0.46 ; 1.05 ; 3.09 ; 6.60 ; 23.08$. $C 2$, R29S3B16. Median firing rates: 0.0; $0.87 ; 2.14 ; 3.53 ; 6.23 ; 10.00$. D1, R3S35B16. Median firing rates: $0.0 ; 0.55 ; 1.26 ; 2.57 ; 4.88 ; 11.43$. D2, R3S37B16. Median firing rates: $0.0 ; 2.00$; $4.00 ; 6.32 ; 9.23 ; 15.00$.

the barrier become much less active and the barrier has no effect on other cells. If this is so, the barrier would be causing a sort of "spatial scotoma," with a great loss of information about the locale of the barrier. It is plausible to expect other cells to develop firing fields when a barrier is introduced. Maps from the one cell whose field intensified in the presence and neighborhood of a barrier are shown in Figure 11. Comparing Figure 11, $A$ and $B$, it is seen that the field expanded greatly around the barrier. Because of sampling problems, a single positive example is a strong indication that the locale of the barrier is "filled in" by cells that are recruited into the map only when the barrier is present.

\section{Irreversible changes in spatial firing patterns}

Up to now, we have maintained the convenient fiction that spatial firing pattern changes have tenure only while the environment is altered. This is justified by the reproducibility of the firing pattern of almost all cells under standard conditions before and after a change in the rat's environment. In 3 cases, however, effects were seen that persisted after the environment was returned to its initial state.

One of the examples (not shown) was seen as a disappearance of the firing field during a session with the cue card rotated, followed by greatly enhanced firing in a different position when another standard session was done. We were convinced by oscilloscope traces of action potentials that the loss of spatial firing did not result from movement of the electrode relative to the cell. The second example (also not illustrated) involved a cardremoval session interpolated between 2 standard sessions. The target neuron ceased to fire about $2 \mathrm{~min}$ into the card-removal session, and continued to be silent everywhere in the small cylinder during the second standard session. Recordings of action potential waveforms lead us to believe that the result was not due to loss of the unit.

The most interesting example of a possible plastic change in spatial firing took place during a rotation experiment that differed from the usual procedure in that the floor paper was not replaced between sessions. The rotation of the firing field after wall plus cue card rotation, seen by comparing Figure 12, $A$ and $B$, is expected from results already presented. When the wall plus cue card were returned to their initial position (Fig. 12C), the bulk of the firing occurred in the predicted location, but the cell continued to fire at a reduced rate in the location appropriate for the rotated case. It is essential to state that the "ghost" left behind during the second standard session was not due to a shift in the predominant location of the field in the course of the session; the same firing pattern was seen in the first and last 8 min of the session. In addition, another standard session done 
B 1
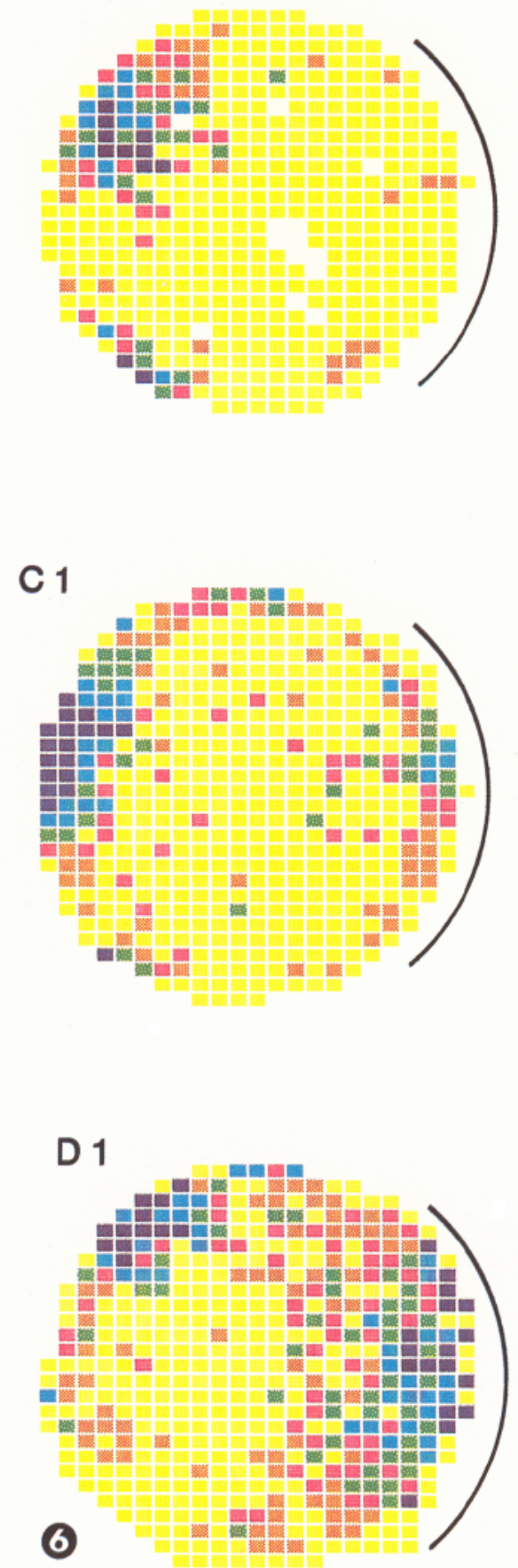

B2

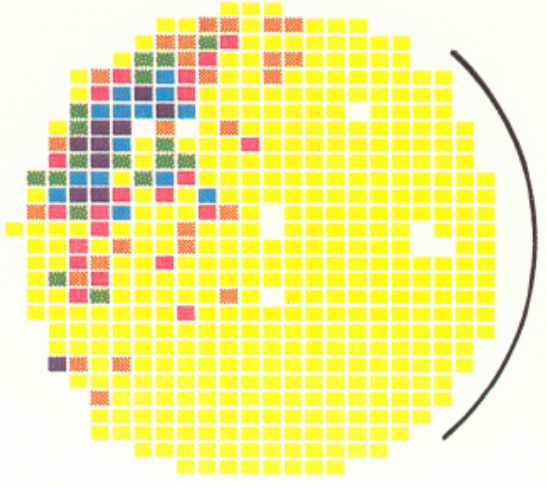

C2
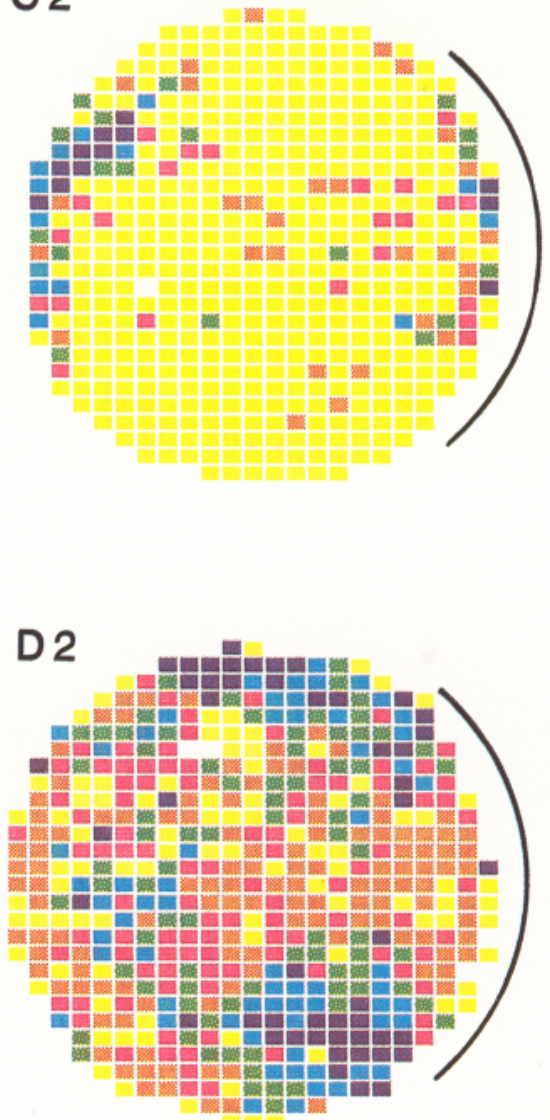

the next day also showed the ghost. We attempted to reproduce this effect on 4 occasions, and saw at most a hint of a ghost only once. Nevertheless, this observation is enough to motivate a search for conditions in which traces of intervening experiences are reliably visible in spatial firing patterns.

\section{Discussion}

Stimulus control over location-specific firing: the cue card and cylinder wall

The great strength and reliability of the spatial firing patterns of place cells when position is the main source of variance in the animal's behavioral state justify the conclusion that place cell firing is location-specific rather than behavior-specific (Muller et al., 1987). For location-specific firing to be reliable, the animal must be able to use available sensory information to distinguish locations from one another. In this section, we consider the types of sensory information that might underlie place cell firing, and the ways that changes in such information can affect place cell firing.

The basic recording chamber was designed so that each of its components (the cylinder wall, the cue card, and the floor paper) could be independently replaced with a copy. Replacing any or all of these components left spatial firing patterns unchanged, 
Figure 7. The responses of 3 place cells to isotropic scaling of the small rectangle. $A$ and $B$ are examples of RF cells; in both cases, the relative position of the firing fields in the 2 rectangles is the same. $C$ shows a UF cell; the 2 fields are unrelated. The rate maps in the large rectangle have been reduced $4: 1 . A$, R2U1; CA3/4. $B$, R8U1; CA1. $C$, R5U1; CA1. Note that examples in this figure and in Figure 6 together show that RF and UF cells are each seen in the $\mathrm{CA} 1$ and $\mathrm{CA} 3 / 4$ pyramidal cell layers of the hippocampus. $A 1, \mathrm{R} 2 \mathrm{~S} 1 \mathrm{~B} 16$. Median firing rates: $0.0 ; 0.47 ; 1.92 ; 4.70$; $8.91 ; 20.00 . A 2$, R2S4B16. Median firing rates: $0.0 ; 1.45 ; 4.62 ; 9.09 ; 17.14$; 28.00. B1, R8S6B8. Median firing rates: $0.0 ; 0.36 ; 1.19 ; 2.65 ; 8.22 ; 20.00$. B2, R8S8B16. Median firing rates: $0.0 ; 0.43$; $1.07 ; 2.35 ; 4.62 ; 9.23$. C1, R5S1B16. Median firing rates: $0.0 ; 0.82 ; 2.30 ; 4.39$; 6.82 ; 10.17. $C 2$, R5S4B16. Median firing rates: $0.0 ; 1.77 ; 4.62 ; 8.57 ; 14.46$; 24.83 .
A 1

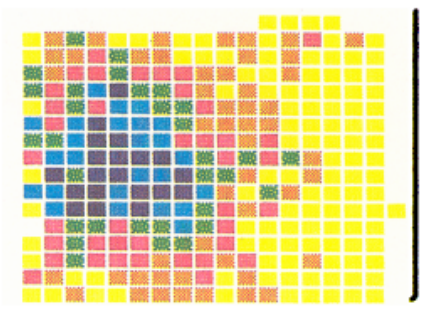

B 1

A 2

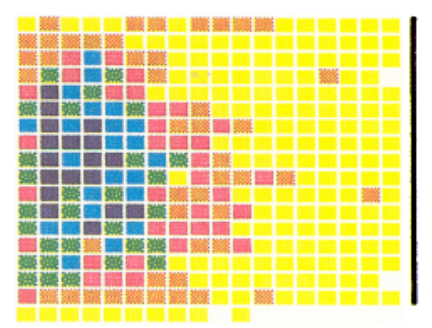

B2

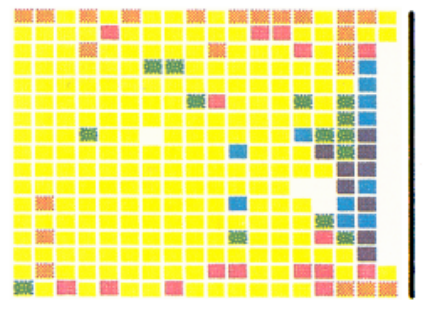

C 1

C2
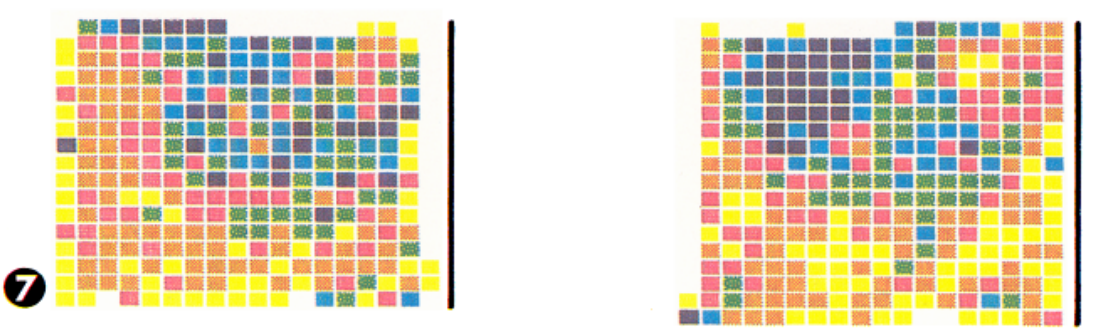

demonstrating the lack of importance of uncontrolled details such as urine spots, blemishes on the cue card, or variations in the texture of the wall. The remaining potential stimuli, the cylinder wall and the cue card, were then tested for salience.
The finding that rotations of the cue card produce equal rotations of firing fields showed that the cue card exerts a form of stimulus control over spatial firing. The finding that substituting a rectangular enclosure for the cylinder alters firing fields beyond

Figure 8. The effect of bisecting the firing field of a place cell with an opaque barrier. $A$ is a standard session in the small cylinder. Before running the session in $B 1$, a rate map of the session in $A$ was printed and used to locate the cell's firing field. The barrier was then put in the small cylinder with one end against the wall and with its length along a radius so as to cut the field in half. The rate map in $B I$ shows that the firing field was greatly attenuated. The full extent of the effect is obscured by autoscaling to assign colors to firing rates. Accordingly, a second rate map of the same session was made, using the breakpoints between colors obtained from the session in $A$. The resulting map (B2) makes it evident that the firing rate was greatly reduced. $C l$ is an autoscaled map for a standard session run after the barrier session; it shows that the barrier effect is reversible. The completeness of the reversibility is confirmed by the map in $C 2$, which was made with the same firing rate categories used in $A$ and $B 2$. R18U2; CA3/4. A, R18S7B16. Median firing rates: $0.0 ; 0.81 ; 2.10 ; 4.90 ; 15.00 ; 43.95 . B 1$, R18S8B16. Median firing rates: $0.0 ; 0.67 ; 0.27$; $1.14 ; 1.88 ; 4.00$. B2, R18S8B16. Median firing rates: scaled from R18S7B16. $C 1$, R18S9B16. Median firing rates: $0.0 ; 0.66 ; 1.76 ; 4.75 ; 12.94 ;$ 33.51. C2, R18S9B16. Median firing rates: scaled from R18S7B16.

Figure 9. The effects of different placements of an opaque barrier on place cell firing. $A$ is a rate map of a standard session. The breakpoints between colors for all the other maps in this figure were taken from the values obtained in $A$. In the session shown in $B$, the opaque barrier bisected the erstwhile firing field and drastically decreased the local firing rate. In $C$, the barrier extended radially inward from the wall at 12 o'clock. In this position, the barrier had little effect on the firing field. In $D$, the barrier was set to touch the part of the field closest to the middle of the apparatus. The field is reduced in size, but the disruption is small compared to the effects of the bisection. In $E$, the barrier was placed on the same radius needed to bisect the field, but its outer end was set about $5 \mathrm{~cm}$ from the wall. This placement was almost as effective as bisection. $F$ is a standard session which shows that the field still appears after the earlier manipulations. It is our impression that the progressive decline in field size seen in maps $A, C$, and $D$ is due to a decreased overall firing rate during the large number of sessions run in a single day on this cell. This impression is supported by the firing rate maps of 2 other standard sessions that were done between the sessions illustrated in $B$ and $C$ and in $E$ and $F$; in each of these, the field size was smaller than in the preceding standard session and larger than in the next standard session. Also not illustrated are 2 other bisection sessions; in each of these, the disruption of spatial firing was at least as great as that shown in $B$. R17U5; CA3/4. $A$, R17S8B16. Median firing rates: $0.0 ; 0.71 ; 3.40 ; 8.28 ; 19.07 ; 33.96 . B$, R17S9B16. Median firing rates: scaled from R17S8B16. $C$, R17S11B16. Median firing rates: scaled from R17S8B16. D, R17S12B16. Median firing rates: scaled from R17S8B16. E, R17S14B16. Median firing rates: scaled from R17S8B16. F, R17S18B16. Median firing rates: scaled from R17S8B16. 
A

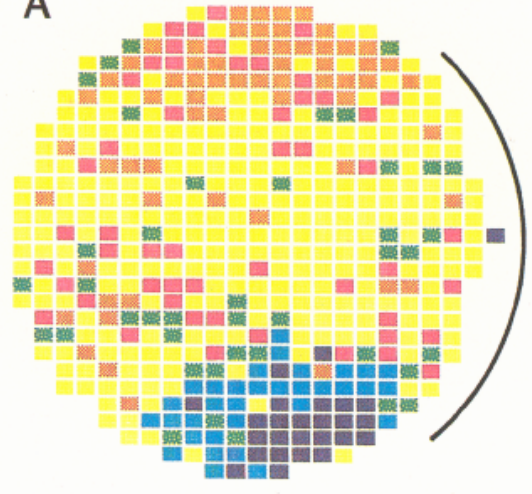

8

A
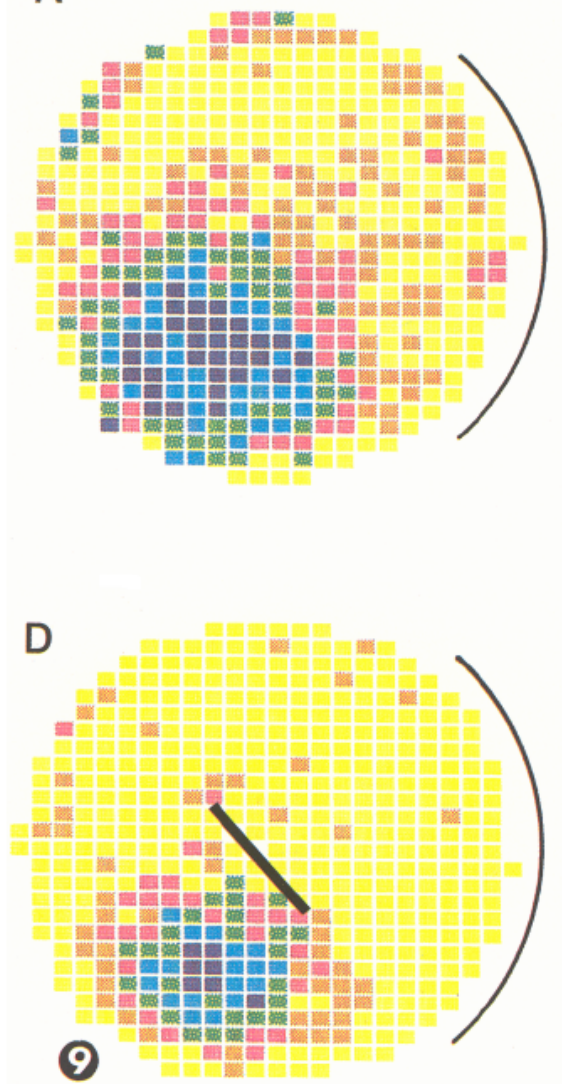

B 1

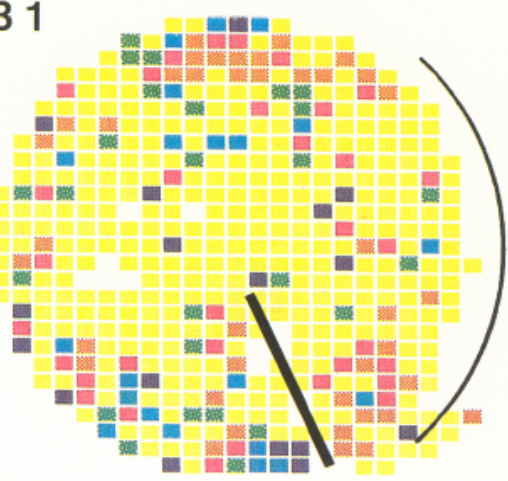

B 2

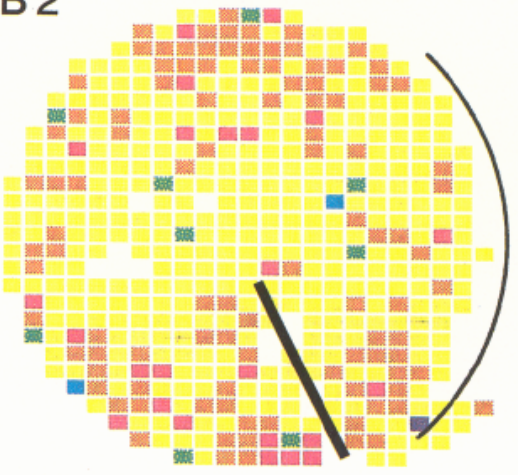

B
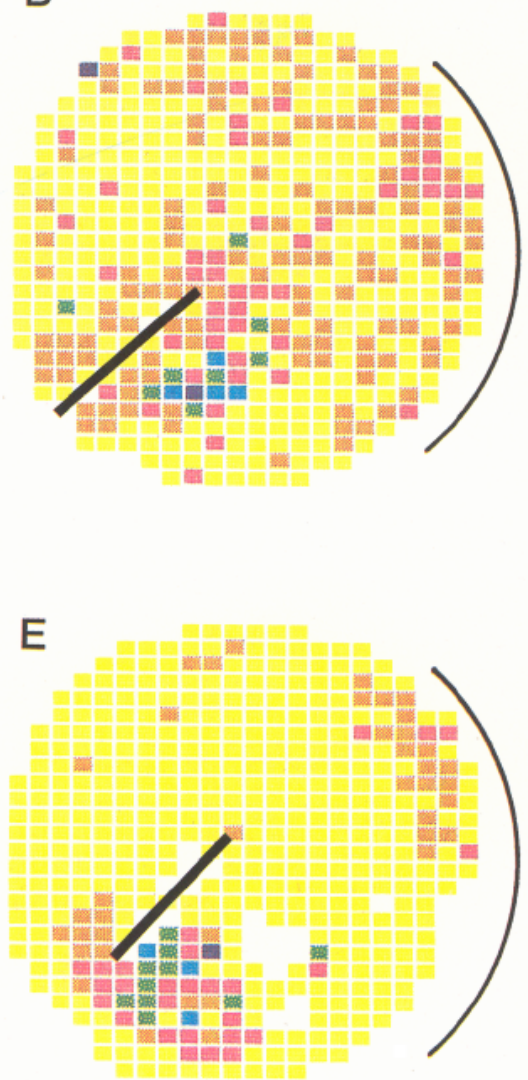

C 1

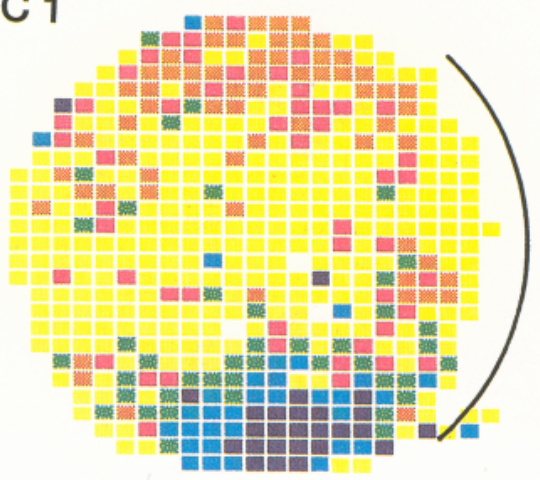

C 2

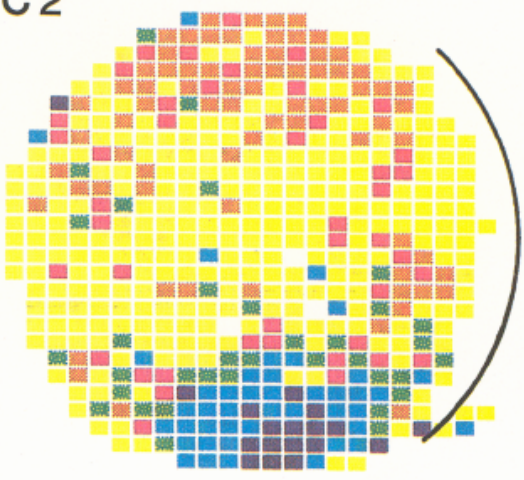

C
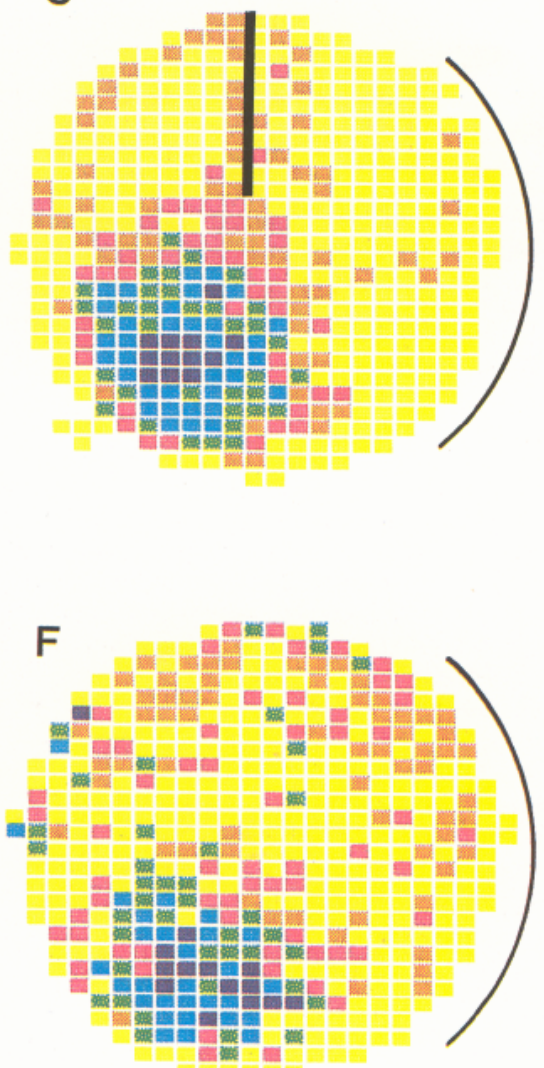
recognition showed that the cylinder wall also exerts a type of stimulus control over spatial firing. With these results, a series of more complex manipulations were performed to ascertain the nature of the control that these stimuli exert over place cell firing.

A convenient way of summarizing the results of complex manipulations of the environment is to compare them to the effects predicted by Zipser's (1985) computational model of place cell firing. Zipser postulates a 2-layer system. The first layer consists of a set of "single feature detectors," each of which is tuned to a position-variant feature of a well-defined stimulus. The second layer is made up of place cells whose activity is imagined to depend on the summed excitatory inputs from a certain set of single feature detectors. It is important, in this model, to distinguish between stimuli and single features of stimuli. For example, the cue card is a stimulus with several potential visual features. A feature detector might respond when the retinal area or width of the card is within a certain range of values, or when there is a certain distance between the card and the rat. With the proper experiments, it should be possible to determine which feature(s) of the stimulus is salient for each recorded place cell. As an aside, it should be noted that the small cylinder has 2-fold symmetry around the diameter that bisects the cue card. Since firing fields in general are not mirror-symmetric, it is possible that the angle between a point on the cue card and the rat is important, or that uncontrolled stimuli are salient. This interesting issue will not be further considered.

The invariance of firing fields (except for a rotation in some cases) in the presence of a half-width cue card suggests that neither the width nor the area of the card is a critical feature; otherwise, fields would be expected to change shape or move closer to the card. Among the remaining possible features are the distance between the animal and the card and the height of the card. In contrast to the ease with which Zipser's (1985) model accounts for half-card experiments, the predictions of the model are out of line with the results of cue-removal experiments. Given the salience of the cue card, the model predicts major changes in the spatial firing pattern after cue removal. Changes that are compatible with the model include cessation of firing, spatially homogeneous firing, or annular firing. There is some degradation of field cohesiveness after card removal, but generally this manipulation leads only to rotation of the intact field to an unpredictable angular position. The restriction of control to a single, abstract field property like angular position by a stimulus of known salience is not compatible with the assertion that field size, shape, and radial position are all codetermined by features of stimuli.

The results of scaling experiments are compatible with the summed-features model. For cells whose fields scale (RF cells), the simplest explanation is that they respond to features that are identical in the 2 cylinders whenever the animal's relative position is the same, or, in other words, that real distance is ignored. For cells whose fields disappear ( $\mathrm{F} / \mathrm{N}$ cells), it is possible that the features that drive each cell no longer simultaneously attain their critical values in any accessible region, so that the summed excitation falls below threshold in the second cylinder. For cells whose firing fields are altered beyond recognition by scaling (UF cells), it could be that the features are simultaneously in the proper range, but in a new part of the apparatus. The $F / N$ and UF cases both imply that at least one of the features (e.g., distance from the cue card) cannot take on the same value when the animal is at the same relative position in the 2 cylinders. A fascinating consequence of interpreting the scaling experiments according to the summed-feature model is that the set of place cells can simultaneously represent the similarity of the 2 cylinders (RF cells), and that they are different in size ( $\mathrm{F} / \mathrm{N}$ and UF cells). In keeping with this interpretation, it should be noted that RF and F/N or UF cells both occur in individual animals (e.g., Fig. 7, $A$ and $D$ ).

To conclude this part of the discussion, it is important to state that the current experiments were not explicitly designed to test Zipser's (1985) model of location-specific firing. We believe it is fair to say that the cue-card manipulations cause firing fields to transform in ways different from those predicted by the model, but more formal tests are certainly in order. In a more positive vein, these results suggest that place cell activity reflects abstract features of the environment, and that the information being processed is better characterized as spatial than sensory.

\section{Variations of place cell firing caused by barriers}

We define a barrier as a vertically oriented, rectangular segment of a plane that makes it impossible for the animal to follow certain paths in the otherwise unobstructed area of the cylinder. A barrier hinders motion but has no other relevant properties, and can therefore be completely described by stating its position and the paths that it forbids. In this section, it is argued that

Figure 10. The effects of different types of barriers on the firing of a place cell. The map in $A$ is from a standard session in the small cylinder; the rest of the maps are scaled according to the breakpoints between colors obtained from this session. In $B$, the opaque barrier bisected the field and drastically reduced local firing. $C$ is a second standard session, which again shows the reversible effects of barriers. In $D$, a transparent (Lucite) barrier the same size as the opaque barrier bisected the field. Its effects were as great as those of the opaque barrier. In $E$, only the lead base used to anchor the barriers was put into the region of the firing field. The effects of the lead base were much less than those caused by either of the barriers. R17U9; CA3/4. A, R17S26B16. Median firing rates: $0.0 ; 0.59 ; 1.53 ; 4.44 ; 12.00 ; 27.12$. B, R17S28B8. Median firing rates: scaled from R17S26B16. C. R17S29B8. Median firing rates: scaled from R17S26B16. D, R17S30B8. Median firing rates: scaled from R17S26B16. E, R17S31B8. Median firing rates: scaled from R17S26B16.

Figure 11. Enhancement of a firing field due to the placement of a barrier. $A$ is a map from a standard session in the small cylinder; a weak field is seen at 5 o'clock. When the transparent barrier was placed to bisect the field, the greatly increased firing seen in $B$ resulted. Another session with the opaque barrier yielded a similar effect on this cell. R42U1; CA3/4. $A, \mathrm{R} 42 \mathrm{~S} 1 \mathrm{~B} 16$. Median firing rates: $0.0 ; 0.28 ; 0.51 ; 0.94 ; 1.42 ; 2.96$. $B$, R42S2B16. Median firing rates: scaled from R42S1B16. When session R42S2B16 was autoscaled, we obtained the following median firing rates for the color categories: $0.0 ; 0.55 ; 1.00 ; 2.94 ; 6.36$. Thus, the barrier caused an increase in field intensity as well as in size and shape.

Figure 12. "Memory" of a wall rotation seen in the firing of a single place cell. $A$ is the map of a standard session run in the small cylinder. In $B$, the walls plus card were rotated $90^{\circ}$ counterclockwise and the firing field rotated an equal amount. In $C$, the walls were returned to their original position. Most of the firing occurred in the expected position, but a "ghost" of the rotated firing field also appeared. As noted in the text, the floor paper was not changed between the sessions illustrated here. R3U2; CA1. $A$, R3S14B16. Median firing rates: $0.0 ; 0.45 ; 1.36 ; 3.18 ; 7.17 ; 15.73$. $B$, R3S16B16. Median firing rates: $0.0 ; 0.48 ; 2.22 ; 4.36 ; 7.50 ; 13.71$. $C$, R3S17B16. Median firing rates: $0.0 ; 0.54 ; 1.80 ; 2.26 ; 4.88 ; 12.41$. 
A

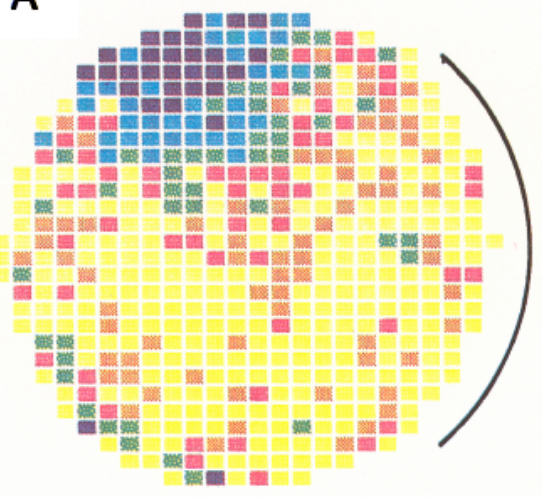

B

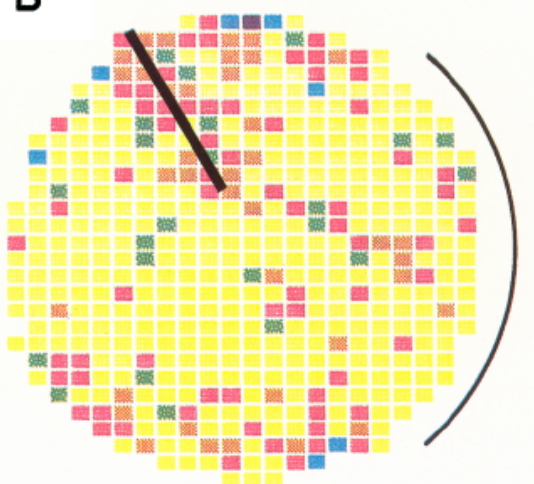

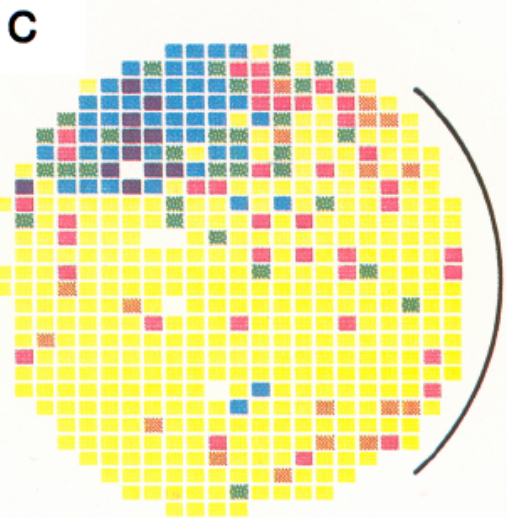

10
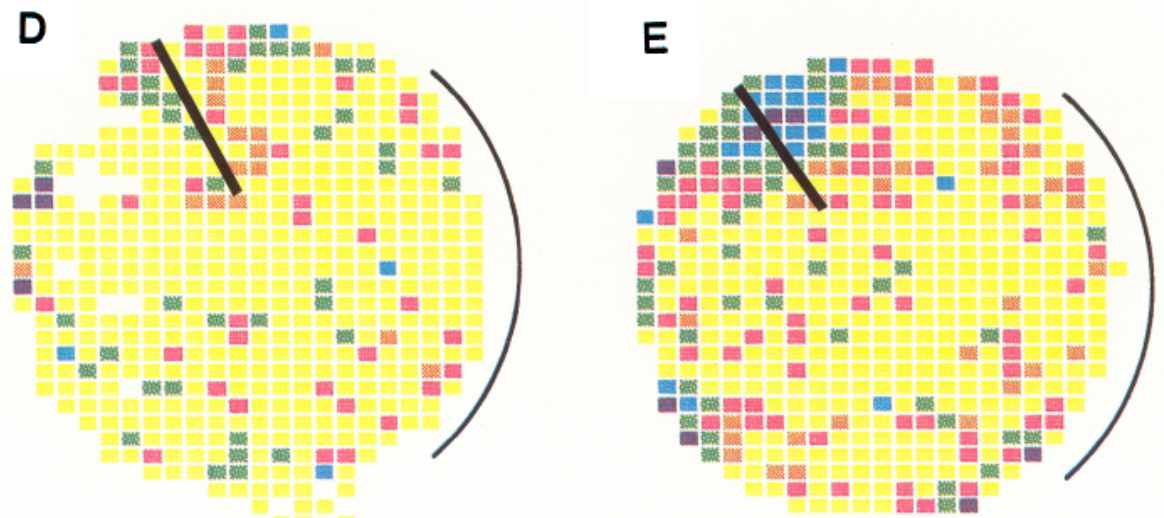

A

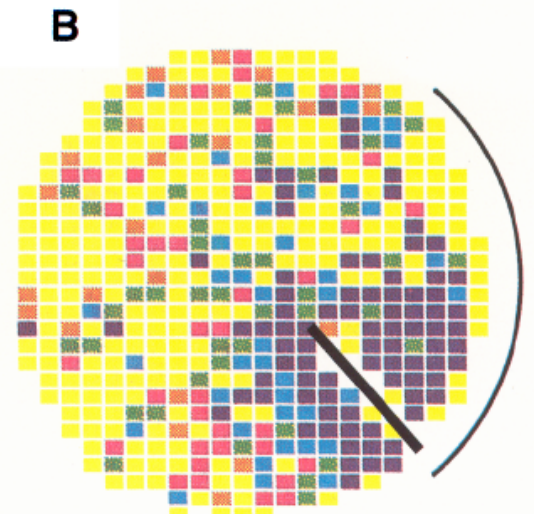

(1)

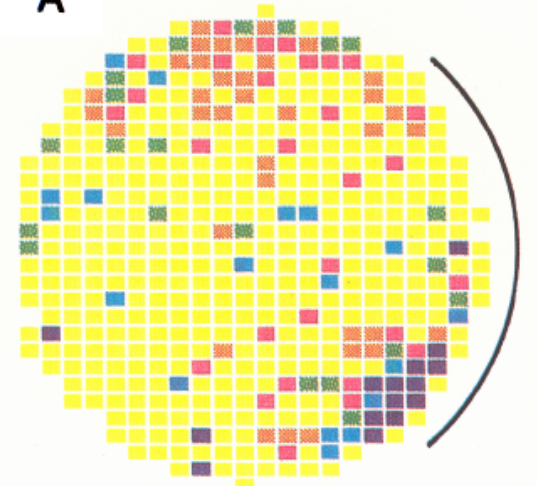

A

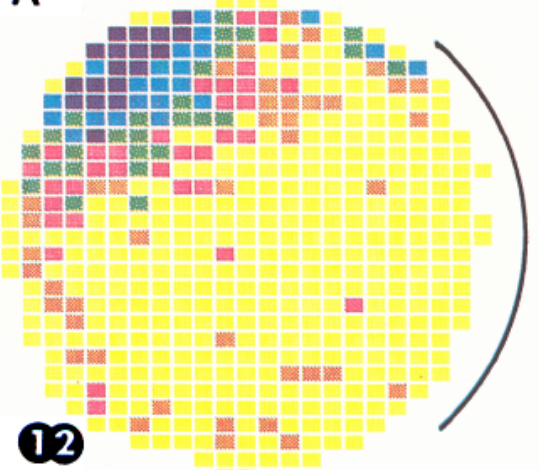

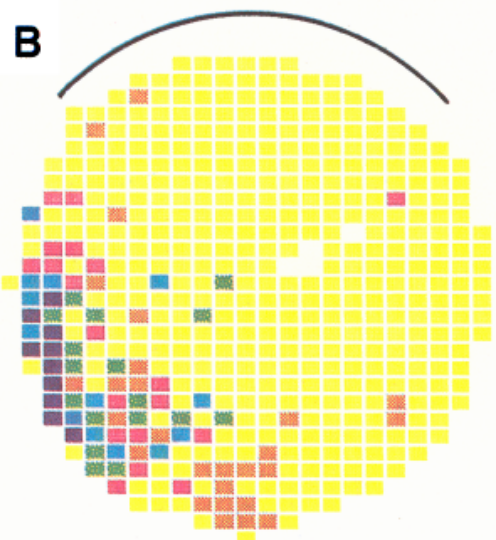

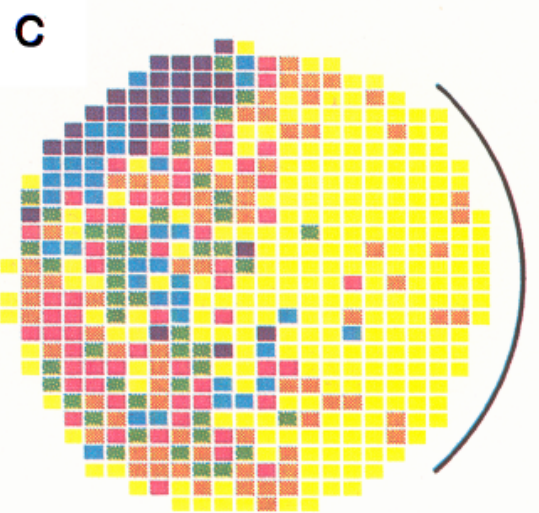


the fiberboard rectangle acts as a barrier, and that the effects it produces on spatial firing are not well explained in terms of simple alterations in the visual appearance of the apparatus nor in terms of simple changes in the animal's behavior. Instead, it is proposed that the changes in place cell firing are associated either with the process of recognizing that the opaque rectangle presents an obstacle to movement or with the efficient, intelligent-looking changes in locomotion that are observed near the barrier.

Sensory interpretations of the barrier effect. The usual result of bisecting a cell's firing field with the fiberboard rectangle is a strong attenuation of the spatial firing of the target neuron. To ascribe this effect to the obstruction created by the opaque rectangle, it must be shown that other rectangles of similar dimensions also cause firing field intensity to weaken. It is impractical to test every possible substitute in this way, but if the effect is seen when an "extremely" different rectangle is used, it is reasonable to suppose that less drastic changes would work the same way. The Plexiglas rectangle was chosen for comparison with the opaque rectangle because of its very different visual appearance, and because it modifies the appearance of the rest of the chamber to a very different extent. ${ }^{1}$ The fact that the 2 rectangles have the same effect on spatial firing implies that simple sensory features are unimportant, but is compatible with the notion that the obstruction is fundamental. The alternative, that the mere presence of an object bisceting the ficld is sufficient to reduce spatial firing, is ruled out by the continued firing when the lead base (used to anchor the rectangles) is used alone. From these observations, it is concluded that place cells may well be responding to the opaque rectangle as an object with spatial properties, and are not reacting to it as a collection of raw sensory features.

The preceding inductive argument is weakened by the use of only 2 additional probes; clearly the range of barriers tested should be extended, especially since other "extremely" different candidates for a substitution experiment exist. In particular, it would be interesting to see the effects of a 2-sided mirror, and of a piece of chicken wire that the animal could climb. Nevertheless, the ability of the Plexiglas to substitute for the fiberboard is striking. Note, also, that this substitution is preferable to others in the sense that the stimulus situation with the Plexiglas rectangle and the lead base are as visually similar to each other as possible, given that one is a potential barrier and the other is not.

Motoric interpretations of the barrier effect. Inserting a barrier into the recording chamber has behavioral as well as sensory consequences, so it is important to see how the barrier effect could arise from shifts in the animal's activity. One possibility is that the behavior is altered everywhere in the apparatus, so that, for instance, the total amount of time spent walking is reduced. This sort of change would be expected to occur for any barrier position and therefore cannot account for the disruption of fields near the barrier and the constancy of fields far away. A second possibility is that the animal's behavioral repertoire shifts in the region local to the barrier, so that the spatial firing

\footnotetext{
${ }^{1}$ The ability of an opaque barrier to reduce the intensity of fields in its neighborhood might be a direct effect of the altered stimulus configuration in the region of the field. The critical stimulus changes are most likely either tactile or visual in origin, because of the nature of the barrier. It is hard to conclude, however, that tactile stimuli are significant, since the suppression of spatial firing extends well away from the barrier. On these grounds, we restrict further considerations to visual stimuli.
}

changes are due to the emission of sniffing or rearing near the barrier. This possibility cannot currently be rejected, but several arguments militatc against it: (1) With repeated exposures to the barrier, both the amount of time spent in its vicinity and the amount of exploratory behavior observed there decrease, whereas there is no tendency of the erstwhile firing field to reappear. (2) There is still a great deal of walking near the barrier, but it is no longer associated with firing. (3) The lead base alone elicits local exploratory behavior, but does not produce attenuation of spatial firing. Thus, a conventional analysis in which behavior is described as a time series of activities such as eating, rearing, or walking does not seem to easily account for the barrier effect.

A major gap in the conventional description of behavior is the lack of attention paid to the fact that locomotor activities, such as walking, carry the animal from one place to another. Thus, walking is commonly treated as a monolithic behavior, and its consequence of moving the animal may be completely neglected; tasks that require the animal to walk to a goal selected on the basis of a sensory discrimination are often called "nonspatial." When the opaque barrier is introduced, however, there are obvious changes in locomotion in its vicinity. In particular, the animal is often seen to walk parallel to the barrier and to always avoid paths that would carry it into the barrier, even during the first exposure. Thus, even if the total amount of time spent walking is constant, it is possible that the altered place cell firing is correlated with these efficient locomotor changes near the barrier. This possibility is attractive, since other assumptions are not required to explain the ability of the transparent barrier to weaken firing fields, the lack of effect of the lead base, or the ineffectiveness of barriers far from the field. The intensified firing of some cells with fields near the barrier suggests that there is no loss of information about the locality of the barrier; the new information may be what is required to restrict the local paths to those that keep the rat from crashing into the barrier.

Summary of the barrier effect. We conclude that the "barrier recognition" and "locomotor change" interpretations of the effect of barriers on place cell firing are viable, high-level alternatives to simpler sensory or behavioral interpretations. From the current data, it is impossible to decide whether the shift in place cell firing is better associated with the recognition or the locomotor interpretation, since both barrier recognition and locomotor changes are expected each time a barrier is inserted. Note, however, that both interpretations are strongly spatial in flavor; the recognition of an obstacle and the ability to reliably avoid it during locomotion imply an abstract representation of the local structure of the environment. In the next section, we consider the possibility that it may be more appropriate to treat barrier recognition and locomotor change as different aspects of a single process, rather than as mutually exclusive behaviors.

\section{Speculations on kinematics}

Kinematics is the study of possible motions. Given a system with a certain number of internal degrees of freedom, which is subject to external constraints, a kinematic analysis can produce a list of the possible states of the system and of allowed trajectories that carry the system from one state to another. We would like to suggest that place cell firing represents a solution to the kinematic problem that the animal faces in getting around in its surroundings.

In this context, it is useful to distinguish between "marker" 
and "structural" stimuli. A marker stimulus provides positional information that may help the animal locate itself, but does not limit the animal's motions. By contrast, a structural stimulus constrains the animal's motions. In the present experiments, the cue card is the only strong marker stimulus, whereas the walls and barriers are structural stimuli.

The main appeal of the kinematic hypothesis comes from the differential effects of wall, barrier, and cue-card manipulations on place cell firing. Changing the shape of the apparatus wall changes the possible trajectories, and is expected to have a major effect on the kinematic solution. The inability to predict spatial firing patterns across changes in apparatus shape is thus taken to mean that an entirely new kinematic solution has been found. Enlarging the apparatus affects trajectory length only, and therefore might be expected to have a less profound effect on the kinematic solution than does shape change. The existence of cells whose fields scale in response to apparatus scaling is taken to mean that the kinematic solutions for similarly shaped apparatuses have much in common. Inserting a barrier mainly changes trajectories in the vicinity of the barrier. The fact that barriers mainly affect nearby firing fields is taken to mean that the new kinematic solution differs from the original only near the barrier. Manipulations of the cue card do not change the available trajectories. The preservation of firing fields after manipulations of the cue card is taken to mean that the kinematic solution has not changed.

We conclude that it is at least not difficult to systematically describe these main results by means of an assumption that the set of place cells is calculating a solution to the kinematic problem. In addition, the idea can be qualitatively tested by investigating place cell responses to movable objects put into the environment, since these are distinguishable on kinematic grounds from walls, barriers, and markers. The prediction is that small, mobile objects will not change place cell firing since they do not constrain the animal's motions. It is possible that ordinary small objects and self-mobile objects (e.g., other rats) will have to be treated differently, but it is encouraging that the kinematic hypothesis has predictive power.

\section{Spatial hypothesis of hippocampal function: place cells and hippocampal lesions}

O'Keefe and Nadel (1978) proposed that the hippocampus is a crucial part of a system that allows rats to get around in their environment in ways that satisfy intuitive notions of efficiency. Informally, it can be imagined that this system allows rats to take shortcuts when they become available, to get to goals from starting points not previously encountered, and so on. A key feature of this theory is that such behavioral capacities are based on a maplike representation of the environment. In the tradition of Tolman (1948), the flexibility of behavior is thought to depend on a neural network that reflects spatial relationships (angles, distances, etc.) among parts of the environment, and that can signal the rat's position within the environment.

The O'Keefe and Nadel (1978) argument that the hippocampus processes spatial information was based on the existence of place cells and on the behavioral consequences of hippocampal lesions. From an extensive review of the literature, O'Keefe and Nadel made the empirical generalization that the effects of such lesions are secondary to the loss of a system that allows efficient, intelligent solutions to problems that involve getting from one place to another. They noted that animals with hippocampal lesions can sometimes solve such problems, but that the strat- egies they use are not as clever. The review can be faulted for its somewhat procrustean flavor, but the variety of observations that fit the spatial hypothesis is impressive.

Subsequently, a number of studies designed to test the spatial hypothesis using the Morris water maze task (Morris, 1980, 1983; Morris et al., 1982; Sutherland et al., 1982) have confirmed the importance of the hippocampus in spatial problem solving. Kolb et al. (1983) showed that frontal or parietal lesions also lead to deficits in the swimming task; but even if the spatial function is shared among several parts of the brain, it seems clear that recent behavioral/lesion studies support the scheme of O'Keefe and Nadel (1978). The fact that hippocampal lesions affect spatial performance is critical for interpreting place cell experiments, since it suggests that place cells are directly involved in processing spatial information. Clearly, it is essential to work out how information supplied to place cells is organized and how place cell firing influcnces other ncurons, but the lesion work supports the notion that location-specific firing is not an epiphenomenon.

The place cell results presented in this and the previous paper (Muller et al., 1987) complement recent lesion results in that they strongly confirm a basic aspect of the spatial theory of hippocampal function (O'Keefe and Nadel, 1978). By providing a clearer picture of the spatial firing properties of place cells, the current results have allowed the appropriateness of the term "place cell" to be better scrutinized. In no case was there a strong discrepancy between observed properties and expectations based on the spatial theory, although the inaccuracy of the animal's calculated position near walls and the variation of firing field shape near walls are unexpected from the original statement of the theory (Muller et al., 1987). The nature of environmental control over place cell firing that is described in this paper also fits the spirit of the original theory, although the details comprise new information. Our general conclusion is therefore that the spatial theory of O'Keefe and Nadel (1978) captures a crucial aspect of hippocampal function. We believe that any attempt to state the general function of the rat hippocampus can at best be incomplete if it does not predict the existence and properties of place cells.

\section{References}

Hill, A. J., and P. J. Best (1981) Effects of deafness and blindness on the spatial correlates of hippocampal unit activity in the rat. Exp. Neurol. 74: 204-217.

Kolb, B., R. J. Sutherland, and I. Q. Wishaw (1983) A comparison of the contributions of the frontal and parietal association cortex to spatial localization in rats. Behav. Neurosci. 97: 13-27.

Kubie, J. L., and J. B. Ranck, Jr. (1983) Sensory-behavioral correlates of individual hippocampal neurons in three situations: Space and context. In Neurobiology of the Hippocampus, W. Seifert, ed., pp. 433-447, Academic, New York.

McNaughton, B. L., J. O'Keefe, and C. A. Barnes (1983) The stereotrade: A new technique for simultaneous isolation of several single units in the central nervous system from multiple unit records. $J$. Neurosci. Methods 8: 391-397.

Morris, R. G. M. (1980) Spatial localization does not require the presence of local cues. Learn. Mot. 12: 239-260.

Morris, R. G. M. (1983) An attempt to dissociate "spatial-mapping" and "working-memory" theories of hippocampal function. In Neurobiology of the Hippocampus, W. Seifert, ed., pp. 405-432, Academic, New York.

Morris, R. G. M., P. Garrud, J. N. P. Rawlins, and J. O'Keefe (1982) Place navigation impaired in rats with hippocampal lesions. Nature 297: 681-683.

Muller, R. U., J. L. Kubie, and J. B. Ranck, Jr. (1987) Spatial firing patterns of hippocampal complex-spike cells in a fixed environment. J. Neurosci. 7: 1935-1950. 
O'Keefe, J. (1976) Place units in the hippocampus of the freely-moving rat. Exp. Neurol. 51: 78-109.

O'Keefe, J., and D. H. Conway (1978) Hippocampal place units in the freely moving rat: Why they fire where they fire. Exp. Brain Res. 31: $573-590$.

O'Keefe, J., and L. Nadel (1978) The Hippocampus as a Cognitive Map, Clarendon Press, Oxford, UK

Olton, D., M. Branch, and P. Best (1978) Spatial correlates of hippocampal unit activity. Exp. Neurol. 58: 387-409.

Ranck, J. B., Jr. (1973) Studies on single neurons in dorsal hippocampal formation and septum in unrestrained rats. Exp. Neurol. 41: 461555.

Ranck, J. B., Jr., J. L. Kubie, S. F. Fox, S. M. Wolfson, and R. U.
Muller (1983) Single neuron recording in behaving mammals: Bridging the gap between cell biological variables and sensory-behavioral variables. In Behavioral Contributions to Brain Research. T. Robinson, ed., Oxford U. P., Oxford, UK.

Sutherland, R. J., B. Kolb, and I. Q. Wishaw (1982) Spatial mapping: Definitive disruption by hippocampal or medial frontal damage in the rat. Neurosci. Lett. 31: 271-276.

Tolman, E. C. (1948) Cognitive maps in rats and men. Psychol. Rev. 55: $189-208$

Walker, H. M., and J. Lev (1953) Statistical Inference, pp. 249-255, Holt, Rinehart and Winston, New York.

Zipser, D. (1985) A computational model of hippocampal place fields. Behav. Neurosci. 99: 1006-1017. 